CRYSTALLOGRAPHIC COMMUNICATIONS

ISSN 2056-9890

Received 27 September 2021

Accepted 15 October 2021

Edited by G. Diaz de Delgado, Universidad de Los Andes, Venezuela

Keywords: crystal structure; piperazinium salts; benzoate anion; biological activity.

CCDC references: $2115865 ; 2115864$; 2115863

Supporting information: this article has supporting information at journals.iucr.org/e
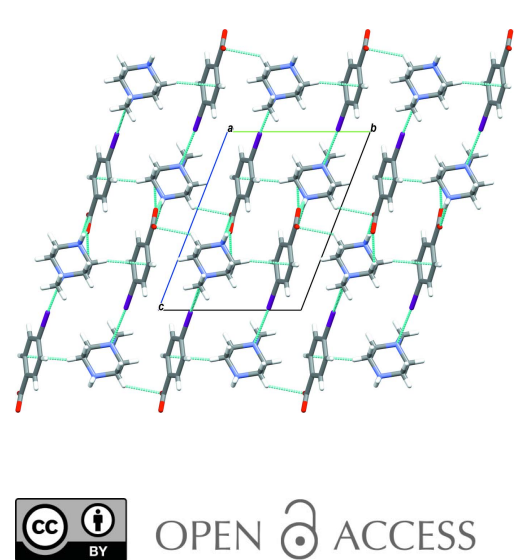

OPEN $\odot$ ACCESS

\section{Crystal structure studies of 4-ethylpiperazin-1-ium 3,5-dinitrobenzoate, 4-methylpiperazin-1-ium 3,5- dinitrobenzoate and 4-methylpiperazin-1-ium 4-iodobenzoate}

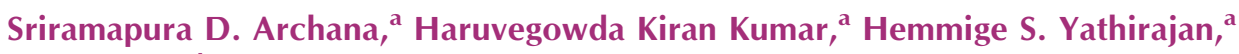
Sabine Foro, ${ }^{b}$ Mohammed S. M. Abdelbaky ${ }^{c}$ and Santiago Garcia-Granda ${ }^{\text {** }}$

${ }^{\mathbf{a}}$ Department of Studies in Chemistry, University of Mysore, Manasagangotri, Mysore-570 006, India, ${ }^{\mathbf{b}}$ Institute of Materials Science, Darmstadt University of Technology, Alarich-Weiss-Strasse 2, D-64287 Darmstadt, Germany, and ${ }^{\mathbf{c}}$ Department of Physical and Analytical Chemistry, Faculty of Chemistry, Oviedo University-CINN, Oviedo 33006, Spain. *Correspondence e-mail: sgg@uniovi.es

As part of our ongoing investigation on the chemical and biological properties of piperazinium salts, we synthesized three novel compounds: 1-ethylpiperazinium 3,5-dinitrobenzoate (I), 1-methylpiperazinium 3,5-dinitrobenzoate (II) and 1-methylpiperazinium 4-iodobenzoate (III). The crystal structures of these compounds are built up of organic layers formed by the strong connection between the molecules by hydrogen bonds of type $\mathrm{N}-\mathrm{H} \cdots \mathrm{O}$. These layers are linked through $\mathrm{N}-\mathrm{H} \cdots \mathrm{O}$ hydrogen bonds and $\mathrm{C}-\mathrm{H} \cdots \mathrm{O}$ interactions or $\mathrm{C}-$ $\mathrm{I} \cdots \mathrm{N}$ halogen bonding, leading to the formation of a three-dimensional network.

\section{Chemical context}

Piperazines and substituted piperazines are important pharmacophores that can be found in many biologically active compounds across a number of different therapeutic areas (Berkheij, 2005) such as antifungal (Upadhayaya et al., 2004), anti-bacterial, anti-malarial and anti-psychotic agents (Choudhary et al., 2006). A valuable insight into recent advances on antimicrobial activity of piperazine derivatives has been reported (Kharb et al., 2012).

Piperazines are among the most important building blocks in today's drug discovery efforts and are found in biologically active compounds across a number of different therapeutic areas (Brockunier et al., 2004; Bogatcheva et al., 2006). A review of the current pharmacological and toxicological information for piperazine derivatives is given by Elliott (2011).

1-Ethylpiperazine is used in the synthesis of 2-\{2-methoxy5-[(4-methylpiperazin-1-yl)sulfonyl]phenyl\}-1H-benzo[d]imidazole hydrochloride and 2-\{5-[(4-ethylpiperazin-1-yl)sulfonyl]-2-methoxyphenyl\}-1H-benzo[ $d]$ imidazole hydrochloride as benzimidazole analogs of sildenafil, which is marketed for the treatment of erectile dysfunction (Qandil, 2012). It is also employed as an intermediate in veterinary medicine and serves as a precursor in the preparation of dyes. $N$-Ethyl piperazine is used in the synthesis of enrofloxacin, which is an antibiotic used to treat bacterial infections. It is also used in the synthesis of dyes, agrochemicals and other pharmaceutical compounds. The crystal structures of compounds derived from 1-ethylpiperazine, viz., chlorobis- 
(2-chlorobenzyl)(4-ethylpiperazine-1-dithiocarbamato$\left.\kappa^{2} S, S^{\prime}\right) \operatorname{tin}(\mathrm{IV}) \quad(\mathrm{Li} \& \mathrm{Li}, 2007)$, 1-diphenylmethyl-4-ethylpiperazine-1,4-diium dichloride (Qiao et al., 2010), (S)-3chloro-4-(4-ethylpiperazin-1-yl)-5-[(1R,2S,5R)-2-isopropyl-5methylcyclohexyloxy]furan-2(5H)-one (Fu et al., 2010), 4-\{[5(4-chlorophenyl)-1-(4-fluorophenyl)-1 $H$-pyrazol-3-yl]carbonyl\}- $N$-ethylpiperazine-1-carboxamide (Shahani et al., 2011), 2-[4-(2-methoxyphenyl)piperazin-1-yl]- $N$-(pyridin-2-yl)acetamide (Lu \& Jiang, 2011), $N$-(4-chlorophenyl)-4-ethylpiperazine-1-carboxamide ( $\mathrm{Li}, 2011)$ and trichlorido(1ethylpiperazin-1-ium)cobalt(II) (Dhieb et al., 2014) have been reported.

1-Methylpiperazine is used in the preparation of 2-(4methyl-1-piperazinylmethyl)acrylophenone as an antimicrotubular drug (Mallevais et al., 1984). It is involved in the preparation of 1-(4-methoxyphenyl)-4-methylpiperazine by reaction with 1-chloro-4-methoxy-benzene. It acts as an intermediate in the synthesis of active pharmaceutical ingredients such as ofloxacin, rifampicin, clozapine, sildenafil, trifluoperazine and zopiclone. The crystal structures of 1-methylpiperazine-1,4-diium 4-nitrophthalate(2-) 4-nitrophthalic acid monohydrate (Guo, 2004), (-)-2-methylpiperazin-1-ium perchlorate (Peng, 2010), 1-methylpiperazine-1,4diium dipicrate (Dutkiewicz et al., 2011), 1-methylpiperazine1,4-dium bis(hydrogen oxalate) (Essid et al., 2014), 2-methylpiperazine-1,4-diium bis(hydrogen maleate) (Wecharine $e t$ al., 2015) and 2-methylpiperazine-1,4-diium bis(hydrogen maleate) (Wecharine \& Arto, 2015), have been reported.<smiles>CCN1CCNCC1</smiles>

(I)<smiles>CN1CCNCC1</smiles>

(II)<smiles>CN1CCNCC1</smiles>

(III)

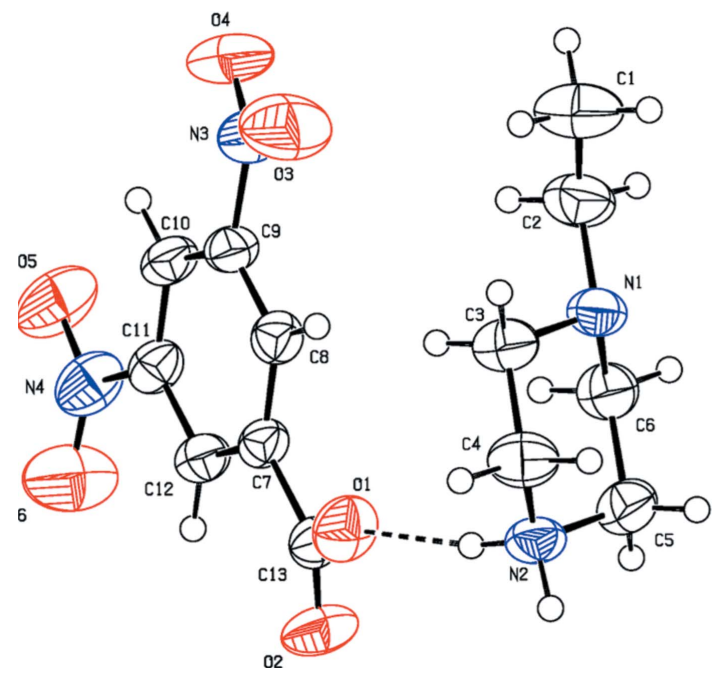

Figure 1

The molecular structure of compound (I), showing the atom labelling. Displacement ellipsoids are drawn at the $50 \%$ probability level.

We have recently reported the crystal structures of some salts of 4-methoxyphenylpiperazine (Kiran Kumar et al., 2019) and also 2-methoxyphenylpiperazine (Harish Chinthal et al., 2020). In view of the importance of piperazines in general and the use of 1-ethyl/methylpiperazine in particular, the present paper reports the crystal structure of salts 1-ethylpiperazinium 3,5-dinitrobenzoate (I), 1-methylpiperazinium 3,5-dinitrobenzoate (II) and 1-methylpiperazinium 4-iodobenzoate (III).

\section{Structural commentary}

The molecular structures of the title salts (I), (II) and (III) are illustrated in Figs. 1, 2 and 3, respectively. The asymmetric unit of compound (I) is composed of one 1-ethylpiperazinium cation and one 3,5-dinitrobenzoate anion while (II) consists of a 1-methylpiperazinium cation and a 3,5-dinitrobenzoate anion. Compound (III) crystallizes with one 1-methylpiperazinium cation and one 4-iodobenzoate anion in the asymmetric unit. In all compounds, the piperazine rings have a
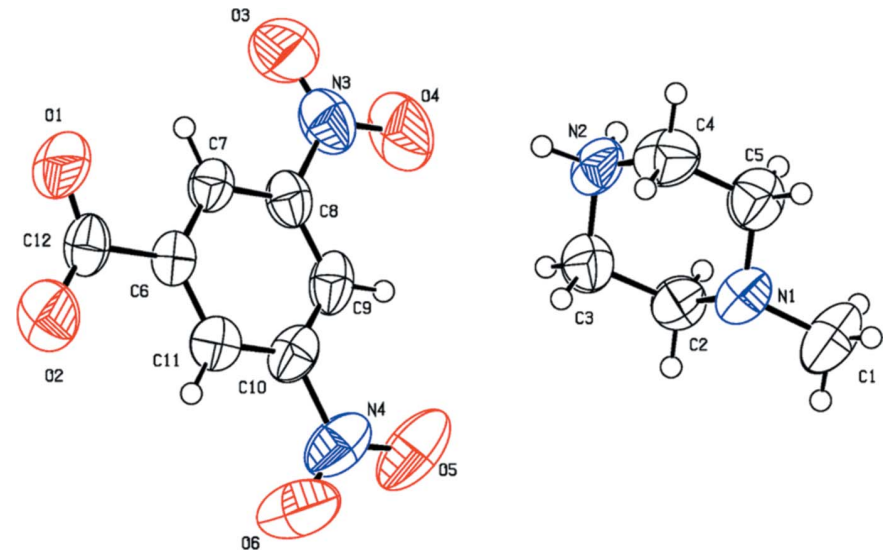

Figure 2

The molecular structure of compound (II), showing the atom labelling. Displacement ellipsoids are drawn at the $50 \%$ probability level. 


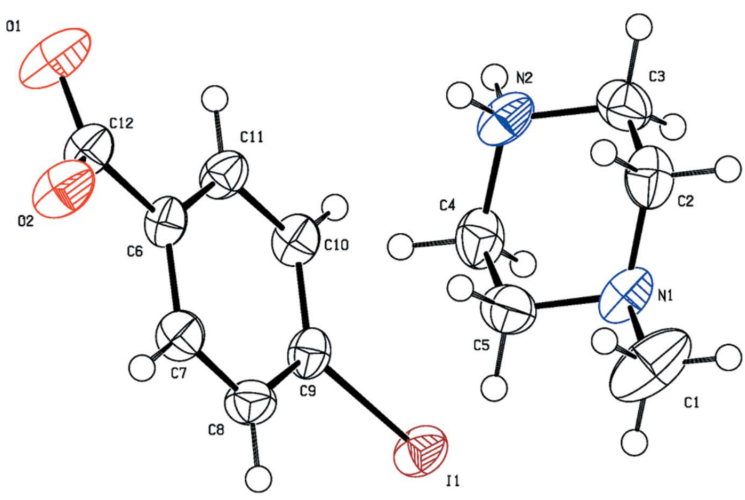

Figure 3

The molecular structure of compound (III), showing the atom labelling. Displacement ellipsoids are drawn at the $50 \%$ probability level.

chair conformation with a positively charged protonated $\mathrm{N}$ atom with a maximum deviation from their mean plane of 0.239 (2), 0.258 (2) and 0.238 (2) $\AA$ at atom N1, for the three title compounds, respectively. The benzene rings are almost

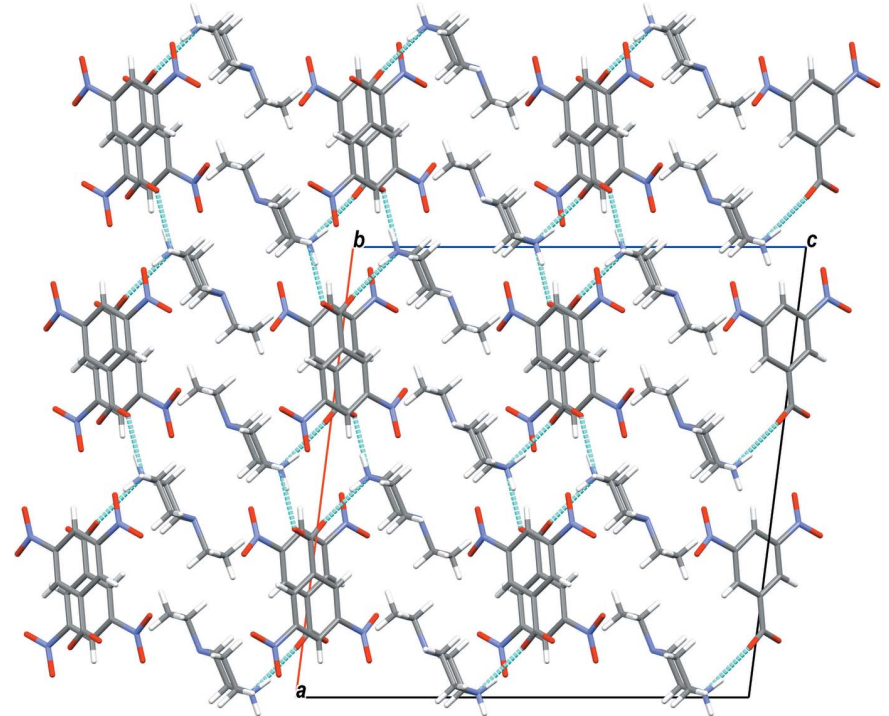

Figure 4

Molecular packing of (I) with hydrogen bonding shown as dashed lines.

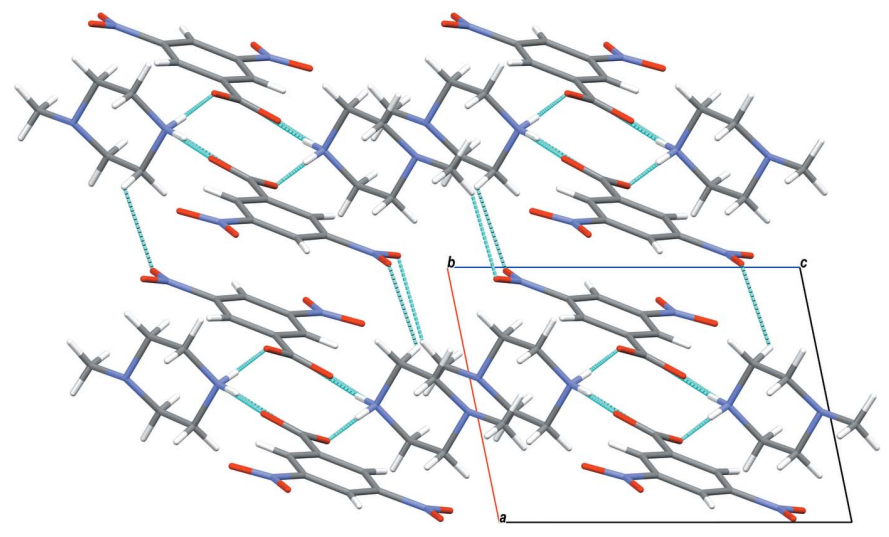

Figure 5

Molecular packing of (II) with hydrogen bonding shown as dashed lines..
Table 1

Hydrogen-bond geometry $\left(\AA,^{\circ}\right)$ for (I).

\begin{tabular}{lllll}
\hline$D-\mathrm{H} \cdots A$ & $D-\mathrm{H}$ & $\mathrm{H} \cdots A$ & $D \cdots A$ & $D-\mathrm{H} \cdots A$ \\
\hline $\mathrm{N} 2-\mathrm{H} 21 \cdots \mathrm{O} 1$ & $0.91(2)$ & $1.88(2)$ & $2.768(2)$ & $168(3)$ \\
$\mathrm{N} 2-\mathrm{H} 22 \cdots \mathrm{O} 2^{\mathrm{i}}$ & $0.92(2)$ & $1.77(2)$ & $2.684(2)$ & $171(3)$ \\
\hline
\end{tabular}

Symmetry code: (i) $-x,-y,-z$.

Table 2

Hydrogen-bond geometry ( $\mathrm{A},{ }^{\circ}$ ) for (II).

\begin{tabular}{lllll}
\hline$D-\mathrm{H} \cdots A$ & $D-\mathrm{H}$ & $\mathrm{H} \cdots A$ & $D \cdots A$ & $D-\mathrm{H} \cdots A$ \\
\hline $\mathrm{N} 2-\mathrm{H} 21 \cdots \mathrm{O} 2^{\mathrm{i}}$ & $0.91(2)$ & $1.82(2)$ & $2.728(2)$ & $175(2)$ \\
$\mathrm{N} 2-\mathrm{H} 22 \cdots \mathrm{O} 1^{i i}$ & $0.91(2)$ & $1.78(2)$ & $2.691(2)$ & $172(2)$ \\
\hline
\end{tabular}

Symmetry codes: (i) $x, y+1, z$; (ii) $-x+1,-y,-z+1$.

Table 3

Hydrogen-bond geometry $\left(\AA,^{\circ}\right)$ for (III).

$\mathrm{Cg} 2$ is the centroid of the C6-C11 ring.

\begin{tabular}{lllll}
\hline$D-\mathrm{H} \cdots A$ & $D-\mathrm{H}$ & $\mathrm{H} \cdots A$ & $D \cdots A$ & $D-\mathrm{H} \cdots A$ \\
\hline $\mathrm{C} 3-\mathrm{H} 3 A \cdots \mathrm{O} 2^{\mathrm{i}}$ & 0.97 & 2.56 & $3.272(3)$ & 130 \\
$\mathrm{C} 5-\mathrm{H} 5 A \cdots \mathrm{O} 1^{\mathrm{ii}}$ & 0.97 & 2.56 & $3.469(3)$ & 156 \\
$\mathrm{~N} 2-\mathrm{H} 21 \cdots \mathrm{O} 2^{\mathrm{ii}}$ & $0.87(2)$ & $1.83(2)$ & $2.696(3)$ & $172(3)$ \\
$\mathrm{N} 2-\mathrm{H} 22 \cdots \mathrm{O} 1^{\mathrm{iii}}$ & $0.88(2)$ & $1.83(2)$ & $2.700(3)$ & $172(3)$ \\
$\mathrm{C} 4-\mathrm{H} 4 B \cdots C g 2^{\mathrm{iv}}$ & 0.97 & 2.59 & $3.473(3)$ & 152 \\
\hline \multicolumn{2}{c}{ Symmetry codes: (i) $x, y+1, z ;$} & (ii) & $-x+2,-y+1,-z+1 ;$ \\
$-x+1,-y+1,-z+1 ;$ (iv) $-x,-y,-z$. & &
\end{tabular}

planar, with maximum deviations of 0.010 (2), 0.006 (2) and 0.006 (3) $\AA$ at atoms C8, C10 and C8 for (I), (II) and (III) respectively. The substituents of the benzene rings in all compounds are approximately in the same plane and do not deviate significantly from planarity.

\section{Supramolecular features}

In the crystal of (I), the cation and anion are linked by N2$\mathrm{H} 21 \cdots \mathrm{O} 1$ hydrogen bonds, forming layers extending along the

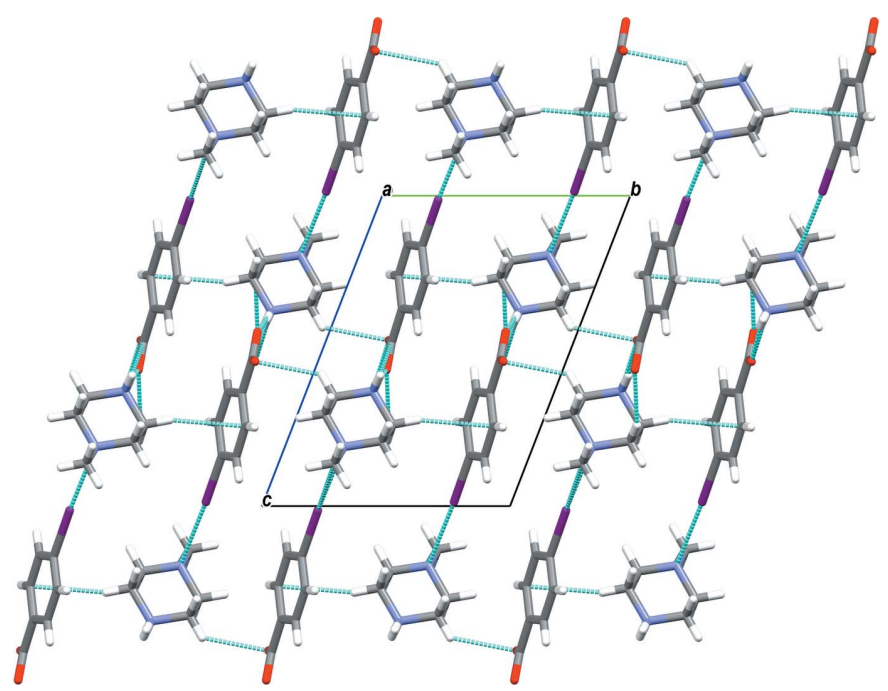

Figure 6

Molecular packing of (III) with hydrogen bonding shown as dashed lines.. 
Table 4

Experimental details.

(I)

\begin{tabular}{|c|}
\hline $\mathrm{C}_{6} \mathrm{H}_{15} \mathrm{~N}_{2}^{+} \cdot \mathrm{C}_{7} \mathrm{H}_{3} \mathrm{~N}_{2} \mathrm{O}_{6}^{-}$ \\
\hline 326.31 \\
\hline Monoclinic, $C 2 / c$ \\
\hline 293 \\
\hline $19.362(1), 8.6279(7), 19.318(1)$ \\
\hline $90,97.261(8), 90$ \\
\hline $3201.3(4)$ \\
\hline 8 \\
\hline Mo $K \alpha$ \\
\hline 0.11 \\
\hline
\end{tabular}

Crystal data

Chemical formula

$M_{\mathrm{r}}$

Crystal system, space group

Temperature (K)

$a, b, c(\AA)$

$\alpha, \beta, \gamma\left({ }^{\circ}\right)$

$V\left(\AA^{3}\right)$

$Z$

Radiation type

$\mu\left(\mathrm{mm}^{-1}\right)$

Crystal size (mm)

$0.46 \times 0.28 \times 0.24$

Data collection

Diffractometer

Absorption correction

$T_{\min }, T_{\max }$

No. of measured, independent and

observed $[I>2 \sigma(I)]$ reflections

$R_{\text {int }}$

$(\sin \theta / \lambda)_{\max }\left(\AA^{-1}\right)$

Oxford Diffraction Xcalibur

Multi-scan (CrysAlis RED; Oxford

Diffraction, 2009)

$0.964,0.974$

$6345,2943,1968$

0.016

0.602

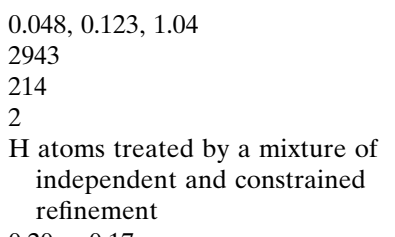

$0.048,0.123,1.04$

$R\left[F^{2}>2 \sigma\left(F^{2}\right)\right], w R\left(F^{2}\right), S$

No. of reflections

No. of parameters

No. of restraints

$\mathrm{H}$-atom treatment

$\Delta \rho_{\max }, \Delta \rho_{\min }\left(\mathrm{e} \AA^{-3}\right)$
(II)

$\mathrm{C}_{5} \mathrm{H}_{13} \mathrm{~N}_{2}{ }^{+} \cdot \mathrm{C}_{7} \mathrm{H}_{3} \mathrm{~N}_{2} \mathrm{O}_{6}{ }^{-}$
312.29
Triclinic, $P \overline{1}$
293
$7.8023(6), 10.3920(8), 10.4770(8)$
$73.578(8), 74.289(8), 71.828(7)$
$758.49(11)$
2
Mo $K \alpha$
0.11
$0.48 \times 0.48 \times 0.44$

Oxford Diffraction Xcalibur

Multi-scan (CrysAlis RED; Oxford

Diffraction, 2009)

$0.948,0.952$

$4819,2774,1935$

0.010

0.602 (III)

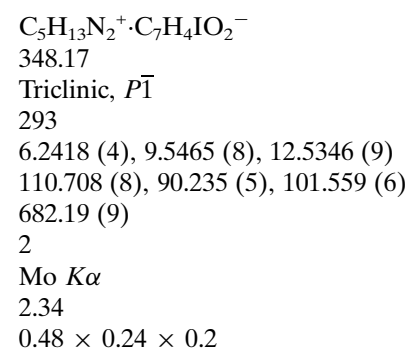

$\begin{array}{ll}0.044,0.129,1.03 & 0.020,0.051,1.11 \\ 2774 & 2492 \\ 206 & 160 \\ 2 & 2 \\ \text { H atoms treated by a mixture of } & \text { H atoms treated by a mixture of } \\ \quad \text { independent and constrained } & \text { independent and constrained } \\ \text { refinement } & \text { refinement } \\ 0.28,-0.15 & 0.37,-0.95\end{array}$

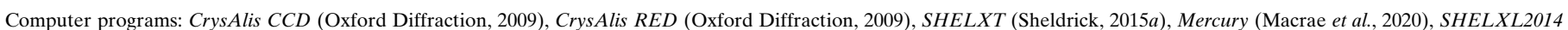
(Sheldrick, 2015b), PLATON (Spek, 2020) and publCIF (Westrip, 2010).

$c$-axis direction. The layers are connected via $\mathrm{N} 2-\mathrm{H} 22 \cdots \mathrm{O} 2$ hydrogen bonds, forming sheets lying parallel to the $a c$ plane (Table 1 and Fig. 4). The crystal structure of compound (II) is built up of $\mathrm{N} 2-\mathrm{H} 21 \cdots \mathrm{O} 2$ and $\mathrm{N} 2-\mathrm{H} 22 \cdots \mathrm{O} 1$ hydrogen bonds that connect the molecules in strong layers along the $c$ axis direction. The layers are linked via weak interactions of the type $\mathrm{C}-\mathrm{H} \cdots \mathrm{O}$, giving a three-dimensional network along the $b$ axis (Table 2 and Fig. 5). The molecules in the crystal of (III) are linked by $\mathrm{N} 2-\mathrm{H} 21 \cdots \mathrm{O} 2, \mathrm{~N} 2-\mathrm{H} 22 \cdots \mathrm{O} 1, \mathrm{C}-\mathrm{H} \cdots \mathrm{O}$ and $\mathrm{C}-\mathrm{H} \cdots \pi$ interactions, forming layers along the $b$ axis. The layers are linked through $\mathrm{C}-\mathrm{I} \cdots \mathrm{N}$ halogen bonding with C9-I1 and $\mathrm{I} 1 \cdots \mathrm{N} 1(1-x, 1-y,-z)$ bond distnces of 2.103 (2) and 3.073 (2) $\AA$, respectively, and bond angle of $174.33(8)^{\circ}$, leading to a three-dimensional structure (Table 3 and Fig. 6).

\section{Database survey}

A search of the Cambridge Structural Database (Version 2020.3.0, last update March 2021; Groom et al., 2016) for the piperazinium cation and benzoate anion involved in the three salts gave 62 hits, 60 of which have branched aromatic substituents either on the piperazinium cation, the benzoate anion or both, that make their structures extremely different from those of the title salts. The other two compounds are quite similar to the title molecules: 4-methylpiperazin-1-ium 2amino-5-iodobenzoate (MAVMEC: Zhu \& Guo, 2005) and 1methylpiperazine-1,4-diium 4-nitrophthalate(2-) 4-nitrophthalic acid monohydrate (IZEFY: Guo, 2004), which share the cationic part and its chair conformation with salts (II) and (III). The crystal structures of the two compounds are based on differently sized rings formed through hydrogen-bond contacts, which then aggregate into a 3D framework.

\section{Synthesis and crystallization}

For the synthesis of (I), a solution of commercially available 1ethylpiperazine $(100 \mathrm{mg}, 0.88 \mathrm{~mol})$ (from Sigma-Aldrich) in methanol $(10 \mathrm{ml})$ was mixed with an equimolar solution of 3,5dinitrobenzoic acid (186.6 mg, $0.88 \mathrm{~mol})$. Compounds (II) and (III) were prepared by the same method in which 1-methylpiperazine $(100 \mathrm{mg}, 1.0 \mathrm{~mol})$ in methanol $(10 \mathrm{ml})$ was mixed with an equimolar solution of 3,5-dinitrobenzoic acid $(212 \mathrm{mg}$, $1.0 \mathrm{~mol}$ ) for (II) or with an equimolar solution of 4-iodobenzoic acid $(248 \mathrm{mg}, 1.0 \mathrm{~mol}$ ) for (III). The corresponding mixtures were stirred for $30 \mathrm{~min}$ at $323 \mathrm{~K}$ and allowed to stand at room temperature. X-ray quality crystals were formed upon slow evaporation in a week time (m.p. 453-455 K, 459-461 K and 410-412 K, respectively). 


\section{Refinement}

Crystal data, data collection and structure refinement details are summarized in Table 4 . The $\mathrm{H}$ atoms bound to $\mathrm{C}$ were positioned with idealized geometry and refined using a riding model with aromatic $\mathrm{C}-\mathrm{H}=0.93 \AA, 0.96 \AA$ (methyl) or $0.97 \AA$ (methylene). The $\mathrm{H}$ atoms of the $\mathrm{N}$ atom were located in a difference map and later restrained to the distance $\mathrm{N}-\mathrm{H}$ $=0.86$ (2) $\AA$. All $\mathrm{H}$ atoms were refined with isotropic displacement parameters set at $1.2 U_{\mathrm{eq}}$ (C-aromatic, $\mathrm{C}$-methylene, $\mathrm{N}$ ) or $1.5 U_{\text {eq }}(\mathrm{C}$-methyl) of the parent atom.

\section{Acknowledgements}

SDA is grateful to the University of Mysore for research facilities. HSY thanks the UGC for a BSR Faculty fellowship for three years. SGG gratefully acknowledges financial support from the Spanish Ministerio de Ciencia e Innovación (PID2020-113558RB-C41) and Gobierno del Principado de Asturias (GRUPIN-ID2018-170).

\section{Funding information}

Funding for this research was provided by: Spanish Ministerio de Ciencia e Innovación (grant No. PID2020-113558RB-C41 to Santiago Garcia-Granda); Gobierno del Principado de Asturias (grant No. GRUPIN-ID2018-170 to Santiago GarciaGranda); University of Mysore (grant to Sriramapura D. Archana); Darmstadt University of Technology (studentship to Hemmige S. Yathirajan).

\section{References}

Berkheij, M., van der Sluis, L., Sewing, C., den Boer, D. J., Terpstra, J. W., Hiemstra, H., Iwema Bakker, W. I., van den Hoogenband, A. \& van Maarseveen, J. H. (2005). Tetrahedron Lett. 46, 2369-2371.

Bogatcheva, E., Hanrahan, C., Nikonenko, B., Samala, R., Chen, P., Gearhart, J., Barbosa, F., Einck, L., Nacy, C. A. \& Protopopova, M. (2006). J. Med. Chem. 49, 3045-3048.

Brockunier, L. L., He, J., Colwell, L. F. Jr, Habulihaz, B., He, H., Leiting, B., Lyons, K. A., Marsilio, F., Patel, R. A., Teffera, Y., Wu, J. K., Thornberry, N. A., Weber, A. E. \& Parmee, E. R. (2004). Bioorg. Med. Chem. Lett. 14, 4763-4766.
Chaudhary, P., Kumar, R., Verma, K., Singh, D., Yadav, V., Chhillar, A. K., Sharma, G. L. \& Chandra, R. (2006). Bioorg. Med. Chem. 14, 1819-1826.

Dhieb, A. C., Janzen, D. E., Rzaigui, M. \& Smirani Sta, W. (2014). Acta Cryst. E70, m166.

Dutkiewicz, G., Samshuddin, S., Narayana, B., Yathirajan, H. S. \& Kubicki, M. (2011). Acta Cryst. E67, o390-o391.

Elliott, S. (2011). Drug Test. Anal. 3, 430-438.

Essid, M., Marouani, H. \& Rzaigui, M. (2014). Acta Cryst. E70, o326o327.

Fu, J.-H., Wang, Z.-Y., Yang, K. \& Mao, C.-X. (2010). Acta Cryst. E66, o2022.

Groom, C. R., Bruno, I. J., Lightfoot, M. P. \& Ward, S. C. (2016). Acta Cryst. B72, 171-179.

Guo, M.-L. (2004). Acta Cryst. C60, o690-0692.

Harish Chinthal, C., Kavitha, C. N., Yathirajan, H. S., Foro, S., Rathore, R. S. \& Glidewell, C. (2020). Acta Cryst. E76, 1779-1793.

Kharb, R., Bansal, K. \& Sharma, A. K. (2012). Der Pharma Chem. 4, 2470-2488.

Kiran Kumar, H., Yathirajan, H. S., Foro, S. \& Glidewell, C. (2019). Acta Cryst. E75, 1494-1506.

Li, J.-Y. \& Li, T.-D. (2007). Acta Cryst. E63, m708-m709.

Li, Y.-F. (2011). Acta Cryst. E67, o2574.

Lu, C. \& Jiang, Q. (2011). Acta Cryst. E67, o223.

Macrae, C. F., Sovago, I., Cottrell, S. J., Galek, P. T. A., McCabe, P., Pidcock, E., Platings, M., Shields, G. P., Stevens, J. S., Towler, M. \& Wood, P. A. (2020). J. Appl. Cryst. 53, 226-235.

Mallevais, M. L., Delacourte, A., Lesieur, I., Lesieur, D., Cazin, M., Brunet, C. \& Luyckx, M. (1984). Biochimie, 66, 477.

Oxford Diffraction (2009). CrysAlis CCD and CrysAlis RED. Oxford Diffraction Ltd, Abingdon, England.

Peng, C.-H. (2010). Acta Cryst. E66, o2114.

Qandil, A. M. (2012). Pharmaceuticals. 5, 460.

Qiao, H.-Y., Xu, S.-H. \& Jiang, H.-X. (2010). Acta Cryst. E66, o1861.

Shahani, T., Fun, H.-K., Vijayakumar, V., Ragavan, R. V. \& Sarveswari, S. (2011). Acta Cryst. E67, o1747-o1748.

Sheldrick, G. M. (2015a). Acta Cryst. A71, 3-8.

Sheldrick, G. M. (2015b). Acta Cryst. C71, 3-8.

Spek, A. L. (2020). Acta Cryst. E76, 1-11.

Upadhayaya, P. S., Sinha, N., Jain, S., Kishore, N., Chandra, R. \& Arora, S. K. (2004). Bioorg. Med. Chem. 12, 2225-2238.

Wecharine, I., Valkonen, A., Rzaigui, M. \& Smirani Sta, W. (2015). Acta Cryst. E71, o193-o194.

Wecharine, I., Valkonen, A., Rzaigui, M. \& Smirani Sta, W. (2015). Acta Cryst. E71, o193-o194.

Westrip, S. P. (2010). J. Appl. Cryst. 43, 920-925.

Zhu, M.-L. \& Guo, M.-L. (2005). Acta Cryst. E61, o3310-o3311. 


\section{supporting information}

Acta Cryst. (2021). E77, 1135-1139 [https://doi.org/10.1107/S2056989021010689]

Crystal structure studies of 4-ethylpiperazin-1-ium 3,5-dinitrobenzoate, 4methylpiperazin-1-ium 3,5-dinitrobenzoate and 4-methylpiperazin-1-ium 4iodobenzoate

Sriramapura D. Archana, Haruvegowda Kiran Kumar, Hemmige S. Yathirajan, Sabine Foro, Mohammed S. M. Abdelbaky and Santiago Garcia-Granda

Computing details

For all structures, data collection: CrysAlis CCD (Oxford Diffraction, 2009); cell refinement: CrysAlis RED (Oxford Diffraction, 2009); data reduction: CrysAlis RED (Oxford Diffraction, 2009); program(s) used to solve structure:

SHELXT (Sheldrick, 2015a); program(s) used to refine structure: SHELXL2014 (Sheldrick, 2015b); molecular graphics: Mercury (Macrae et al., 2020); software used to prepare material for publication: SHELXL2014 (Sheldrick, 2015b), PLATON (Spek, 2020) and publCIF (Westrip, 2010).

4-Ethylpiperazin-1-ium 3,5-dinitrobenzoate (I)

Crystal data

$\mathrm{C}_{6} \mathrm{H}_{15} \mathrm{~N}_{2}^{+} \cdot \mathrm{C}_{7} \mathrm{H}_{3} \mathrm{~N}_{2} \mathrm{O}_{6}$

$M_{r}=326.31$

Monoclinic, $C 2 / c$

Hall symbol: $-\mathrm{C} 2 \mathrm{yc}$

$a=19.362(1) \AA$

$b=8.6279$ (7) $\AA$

$c=19.318(1) \AA$

$\beta=97.261(8)^{\circ}$

$V=3201.3(4) \AA^{3}$

$Z=8$

Data collection

Oxford Diffraction Xcalibur diffractometer

Graphite monochromator

$\omega$ scans

Absorption correction: multi-scan

(CrysAlis RED; Oxford Diffraction, 2009)

$T_{\min }=0.964, T_{\max }=0.974$

6345 measured reflections

Refinement

Refinement on $F^{2}$

Least-squares matrix: full

$R\left[F^{2}>2 \sigma\left(F^{2}\right)\right]=0.048$

$w R\left(F^{2}\right)=0.123$
$F(000)=1376$

$D_{\mathrm{x}}=1.354 \mathrm{Mg} \mathrm{m}^{-3}$

Mo $K \alpha$ radiation, $\lambda=0.71073 \AA$

Cell parameters from 6345 reflections

$\theta=2.6-25.4^{\circ}$

$\mu=0.11 \mathrm{~mm}^{-1}$

$T=293 \mathrm{~K}$

Prism, orange

$0.46 \times 0.28 \times 0.24 \mathrm{~mm}$

2943 independent reflections

1968 reflections with $I>2 \sigma(I)$

$R_{\text {int }}=0.016$

$\theta_{\text {max }}=25.4^{\circ}, \theta_{\text {min }}=2.6^{\circ}$

$h=-23 \rightarrow 23$

$k=-10 \rightarrow 10$

$l=-9 \rightarrow 23$

$S=1.04$

2943 reflections

214 parameters

2 restraints 
0 constraints

Primary atom site location: structure-invariant direct methods

Secondary atom site location: difference Fourier map

Hydrogen site location: mixed
$\mathrm{H}$ atoms treated by a mixture of independent and constrained refinement

$w=1 /\left[\sigma^{2}\left(F_{\mathrm{o}}^{2}\right)+(0.0507 P)^{2}+1.8986 P\right]$ where $P=\left(F_{\mathrm{o}}{ }^{2}+2 F_{\mathrm{c}}{ }^{2}\right) / 3$

$(\Delta / \sigma)_{\max }<0.001$

$\Delta \rho_{\max }=0.20$ e $\AA^{-3}$

$\Delta \rho_{\min }=-0.17$ e $\AA^{-3}$

Special details

Geometry. All esds (except the esd in the dihedral angle between two 1.s. planes) are estimated using the full covariance matrix. The cell esds are taken into account individually in the estimation of esds in distances, angles and torsion angles; correlations between esds in cell parameters are only used when they are defined by crystal symmetry. An approximate (isotropic) treatment of cell esds is used for estimating esds involving l.s. planes.

Fractional atomic coordinates and isotropic or equivalent isotropic displacement parameters $\left(\AA^{2}\right)$

\begin{tabular}{|c|c|c|c|c|}
\hline & $x$ & $y$ & $z$ & $U_{\text {iso }} * / U_{\text {eq }}$ \\
\hline $\mathrm{C} 1$ & $0.18587(15)$ & $0.3364(3)$ & $0.30396(15)$ & $0.0884(9)$ \\
\hline H1A & 0.232834 & 0.339426 & 0.326863 & $0.133 *$ \\
\hline H1B & 0.177587 & 0.424858 & 0.273903 & $0.133 *$ \\
\hline $\mathrm{H} 1 \mathrm{C}$ & 0.154108 & 0.338102 & 0.338302 & $0.133 *$ \\
\hline $\mathrm{C} 2$ & $0.17517(12)$ & 0.1925 & $0.26195(13)$ & $0.0655(7)$ \\
\hline $\mathrm{H} 2 \mathrm{~A}$ & 0.208261 & 0.190584 & 0.228215 & $0.079 *$ \\
\hline $\mathrm{H} 2 \mathrm{~B}$ & 0.184798 & 0.103891 & 0.292568 & $0.079 *$ \\
\hline $\mathrm{C} 3$ & $0.08924(11)$ & $0.2969(3)$ & $0.17179(12)$ & $0.0533(6)$ \\
\hline H3A & 0.12338 & 0.291797 & 0.13919 & $0.064 *$ \\
\hline H3B & 0.092984 & 0.397926 & 0.194012 & $0.064 *$ \\
\hline $\mathrm{C} 4$ & $0.01736(11)$ & $0.2779(3)$ & $0.13276(13)$ & $0.0589(6)$ \\
\hline H4A & -0.017049 & 0.290226 & 0.164733 & $0.071 *$ \\
\hline H4B & 0.009231 & 0.357361 & 0.097152 & $0.071 *$ \\
\hline $\mathrm{C} 5$ & $0.02593(11)$ & 0.0011 & $0.15297(12)$ & $0.0563(6)$ \\
\hline H5A & 0.023563 & -0.099603 & 0.130507 & $0.068 *$ \\
\hline H5B & -0.008248 & 0.003405 & 0.185592 & $0.068 *$ \\
\hline C6 & $0.09733(11)$ & $0.0253(3)$ & $0.19161(12)$ & $0.0538(6)$ \\
\hline H6A & 0.106657 & -0.054243 & 0.227061 & $0.065^{*}$ \\
\hline H6B & 0.131632 & 0.015195 & 0.159377 & $0.065^{*}$ \\
\hline $\mathrm{C} 7$ & $0.22610(9)$ & $0.0114(2)$ & $0.00395(10)$ & $0.0389(5)$ \\
\hline $\mathrm{C} 8$ & $0.25500(10)$ & $0.1070(2)$ & $0.05725(10)$ & $0.0431(5)$ \\
\hline $\mathrm{H} 8$ & 0.226597 & 0.164062 & 0.08315 & $0.052 *$ \\
\hline C9 & $0.32638(10)$ & $0.1168(2)$ & $0.07159(10)$ & $0.0449(5)$ \\
\hline $\mathrm{C} 10$ & $0.37088(10)$ & $0.0395(2)$ & $0.03386(11)$ & $0.0468(5)$ \\
\hline $\mathrm{H} 10$ & 0.418946 & 0.049122 & 0.043855 & $0.056^{*}$ \\
\hline $\mathrm{C} 11$ & $0.34091(9)$ & $-0.0527(2)$ & $-0.01928(11)$ & $0.0442(5)$ \\
\hline $\mathrm{C} 12$ & $0.26950(9)$ & -0.0699 (2) & $-0.03464(10)$ & $0.0428(5)$ \\
\hline H12 & 0.250959 & -0.135397 & -0.070496 & $0.051^{*}$ \\
\hline $\mathrm{C} 13$ & $0.14743(10)$ & -0.0038 & $-0.01245(10)$ & $0.0440(5)$ \\
\hline N1 & $0.10398(8)$ & $0.1771(2)$ & $0.22445(8)$ & $0.0478(5)$ \\
\hline $\mathrm{N} 2$ & $0.00983(8)$ & $0.1234(2)$ & $0.09987(9)$ & $0.0502(5)$ \\
\hline N3 & $0.35665(12)$ & $0.2143(3)$ & $0.13029(11)$ & $0.0657(6)$ \\
\hline
\end{tabular}


supporting information

$\begin{array}{lllll}\mathrm{N} 4 & 0.38632(9) & -0.1369(2) & -0.06158(12) & 0.0612(5) \\ \text { O1 } & 0.11149(7) & 0.0962(2) & 0.01190(8) & 0.0621(5) \\ \text { O2 } & 0.12597(7) & -0.1160(2) & -0.05004(9) & 0.0653(5) \\ \text { O3 } & 0.31860(12) & 0.3056(3) & 0.15461(11) & 0.0980(7) \\ \text { O4 } & 0.41795(10) & 0.1972(3) & 0.15171(10) & 0.0987(7) \\ \text { O5 } & 0.44928(7) & -0.1316(2) & -0.04397(10) & 0.0829(6) \\ \text { O6 } & 0.35960(9) & -0.2085(3) & -0.11194(11) & 0.0962(7) \\ \text { H21 } & 0.0381(14) & 0.116(4) & 0.0660(13) & 0.115^{*} \\ \text { H22 } & -0.0355(10) & 0.114(4) & 0.0790(14) & 0.115^{*}\end{array}$

Atomic displacement parameters $\left(\AA^{2}\right)$

\begin{tabular}{lllllll}
\hline & $U^{11}$ & $U^{22}$ & $U^{33}$ & $U^{12}$ & $U^{13}$ & $U^{23}$ \\
\hline C1 & $0.084(2)$ & $0.088(2)$ & $0.085(2)$ & $-0.0122(16)$ & $-0.0206(15)$ & $-0.0224(17)$ \\
C2 & $0.0594(14)$ & $0.0728(18)$ & $0.0585(14)$ & $-0.0020(12)$ & $-0.0150(11)$ & $0.0000(13)$ \\
C3 & $0.0511(12)$ & $0.0460(13)$ & $0.0597(13)$ & $-0.0019(10)$ & $-0.0053(10)$ & $-0.0028(11)$ \\
C4 & $0.0477(12)$ & $0.0602(15)$ & $0.0652(14)$ & $0.0061(11)$ & $-0.0069(11)$ & $-0.0037(13)$ \\
C5 & $0.0538(13)$ & $0.0543(14)$ & $0.0608(14)$ & $-0.0136(11)$ & $0.0072(11)$ & $-0.0063(12)$ \\
C6 & $0.0546(13)$ & $0.0483(14)$ & $0.0563(13)$ & $-0.0017(10)$ & $-0.0015(10)$ & $0.0037(12)$ \\
C7 & $0.0330(9)$ & $0.0390(11)$ & $0.0437(11)$ & $-0.0016(8)$ & $0.0007(8)$ & $0.0063(10)$ \\
C8 & $0.0431(11)$ & $0.0413(12)$ & $0.0448(11)$ & $-0.0019(9)$ & $0.0053(9)$ & $0.0024(10)$ \\
C9 & $0.0460(11)$ & $0.0423(12)$ & $0.0439(11)$ & $-0.0114(10)$ & $-0.0043(9)$ & $0.0027(10)$ \\
C10 & $0.0331(10)$ & $0.0478(12)$ & $0.0565(13)$ & $-0.0072(9)$ & $-0.0057(9)$ & $0.0094(11)$ \\
C11 & $0.0332(10)$ & $0.0446(12)$ & $0.0547(12)$ & $0.0004(9)$ & $0.0049(9)$ & $0.0032(11)$ \\
C12 & $0.0370(10)$ & $0.0422(12)$ & $0.0479(11)$ & $-0.0039(9)$ & $-0.0002(9)$ & $0.0008(10)$ \\
C13 & $0.0329(10)$ & $0.0542(14)$ & $0.0443(11)$ & $-0.0017(10)$ & $0.0026(9)$ & $0.0081(11)$ \\
N1 & $0.0446(9)$ & $0.0524(11)$ & $0.0437(10)$ & $-0.0038(8)$ & $-0.0048(8)$ & $-0.0025(9)$ \\
N2 & $0.0331(9)$ & $0.0683(13)$ & $0.0477(10)$ & $-0.0049(9)$ & $-0.0013(7)$ & $-0.0090(10)$ \\
N3 & $0.0674(14)$ & $0.0690(15)$ & $0.0579(12)$ & $-0.0222(12)$ & $-0.0030(11)$ & $-0.0041(12)$ \\
N4 & $0.0428(11)$ & $0.0591(13)$ & $0.0833(14)$ & $0.0009(9)$ & $0.0135(10)$ & $-0.0068(12)$ \\
O1 & $0.0415(8)$ & $0.0734(11)$ & $0.0735(11)$ & $0.0069(8)$ & $0.0153(7)$ & $-0.0026(9)$ \\
O2 & $0.0340(8)$ & $0.0748(11)$ & $0.0837(11)$ & $-0.0064(8)$ & $-0.0057(7)$ & $-0.0170(10)$ \\
O3 & $0.1085(17)$ & $0.0929(16)$ & $0.0892(15)$ & $-0.0096(13)$ & $-0.0011(12)$ & $-0.0405(13)$ \\
O4 & $0.0675(12)$ & $0.1305(19)$ & $0.0900(14)$ & $-0.0337(12)$ & $-0.0219(10)$ & $-0.0182(13)$ \\
O5 & $0.0337(9)$ & $0.0929(14)$ & $0.1238(16)$ & $0.0008(9)$ & $0.0167(9)$ & $-0.0136(12)$ \\
O6 & $0.0624(11)$ & $0.1236(18)$ & $0.1026(14)$ & $0.0096(11)$ & $0.0109(10)$ & $-0.0556(14)$ \\
& & & & & & \\
\hline
\end{tabular}

Geometric parameters $\left(\AA,{ }^{o}\right)$

\begin{tabular}{llll}
\hline $\mathrm{C} 1-\mathrm{C} 2$ & $1.484(3)$ & $\mathrm{C} 7-\mathrm{C} 8$ & $1.381(3)$ \\
$\mathrm{C} 1-\mathrm{H} 1 \mathrm{~A}$ & 0.96 & $\mathrm{C} 7-\mathrm{C} 12$ & $1.383(3)$ \\
$\mathrm{C} 1-\mathrm{H} 1 \mathrm{~B}$ & 0.96 & $\mathrm{C} 7-\mathrm{C} 13$ & $1.522(2)$ \\
$\mathrm{C} 1-\mathrm{H} 1 \mathrm{C}$ & 0.96 & $\mathrm{C} 8-\mathrm{C} 9$ & $1.377(3)$ \\
$\mathrm{C} 2-\mathrm{N} 1$ & $\mathrm{C} 8-\mathrm{H} 8$ & 0.93 \\
$\mathrm{C} 2-\mathrm{H} 2 \mathrm{~A}$ & $1.480(3)$ & $\mathrm{C} 9-\mathrm{C} 10$ & $1.370(3)$ \\
$\mathrm{C} 2-\mathrm{H} 2 \mathrm{~B}$ & 0.97 & $\mathrm{C} 9-\mathrm{N} 3$ & $1.473(3)$ \\
$\mathrm{C} 3-\mathrm{N} 1$ & 0.97 & $\mathrm{C} 10-\mathrm{C} 11$ & $1.368(3)$ \\
$\mathrm{C} 3-\mathrm{C} 4$ & $1.453(3)$ & $\mathrm{C} 10-\mathrm{H} 10$ & 0.93
\end{tabular}




\begin{tabular}{|c|c|c|c|}
\hline $\mathrm{C} 3-\mathrm{H} 3 \mathrm{~A}$ & 0.97 & $\mathrm{C} 11-\mathrm{C} 12$ & $1.385(2)$ \\
\hline $\mathrm{C} 3-\mathrm{H} 3 \mathrm{~B}$ & 0.97 & $\mathrm{C} 11-\mathrm{N} 4$ & $1.467(3)$ \\
\hline $\mathrm{C} 4-\mathrm{N} 2$ & $1.476(3)$ & $\mathrm{C} 12-\mathrm{H} 12$ & 0.93 \\
\hline $\mathrm{C} 4-\mathrm{H} 4 \mathrm{~A}$ & 0.97 & $\mathrm{C} 13-\mathrm{O} 1$ & $1.238(2)$ \\
\hline $\mathrm{C} 4-\mathrm{H} 4 \mathrm{~B}$ & 0.97 & $\mathrm{C} 13-\mathrm{O} 2$ & $1.250(2)$ \\
\hline $\mathrm{C} 5-\mathrm{N} 2$ & $1.477(3)$ & $\mathrm{N} 2-\mathrm{H} 21$ & $0.907(17)$ \\
\hline $\mathrm{C} 5-\mathrm{C} 6$ & $1.500(3)$ & $\mathrm{N} 2-\mathrm{H} 22$ & $0.922(17)$ \\
\hline $\mathrm{C} 5-\mathrm{H} 5 \mathrm{~A}$ & 0.97 & $\mathrm{~N} 3-\mathrm{O} 3$ & $1.213(3)$ \\
\hline C5-H5B & 0.97 & $\mathrm{~N} 3-\mathrm{O} 4$ & $1.216(2)$ \\
\hline $\mathrm{C} 6-\mathrm{N} 1$ & $1.454(3)$ & $\mathrm{N} 4-\mathrm{O} 6$ & $1.212(2)$ \\
\hline C6-H6A & 0.97 & $\mathrm{~N} 4-\mathrm{O} 5$ & $1.224(2)$ \\
\hline C6-H6B & 0.97 & & \\
\hline $\mathrm{C} 2-\mathrm{C} 1-\mathrm{H} 1 \mathrm{~A}$ & 109.5 & $\mathrm{C} 8-\mathrm{C} 7-\mathrm{C} 12$ & $119.22(17)$ \\
\hline $\mathrm{C} 2-\mathrm{C} 1-\mathrm{H} 1 \mathrm{~B}$ & 109.5 & $\mathrm{C} 8-\mathrm{C} 7-\mathrm{C} 13$ & $120.47(18)$ \\
\hline $\mathrm{H} 1 \mathrm{~A}-\mathrm{C} 1-\mathrm{H} 1 \mathrm{~B}$ & 109.5 & $\mathrm{C} 12-\mathrm{C} 7-\mathrm{C} 13$ & $120.31(18)$ \\
\hline $\mathrm{C} 2-\mathrm{C} 1-\mathrm{H} 1 \mathrm{C}$ & 109.5 & $\mathrm{C} 9-\mathrm{C} 8-\mathrm{C} 7$ & $119.24(19)$ \\
\hline $\mathrm{H} 1 \mathrm{~A}-\mathrm{C} 1-\mathrm{H} 1 \mathrm{C}$ & 109.5 & $\mathrm{C} 9-\mathrm{C} 8-\mathrm{H} 8$ & 120.4 \\
\hline $\mathrm{H} 1 \mathrm{~B}-\mathrm{C} 1-\mathrm{H} 1 \mathrm{C}$ & 109.5 & $\mathrm{C} 7-\mathrm{C} 8-\mathrm{H} 8$ & 120.4 \\
\hline $\mathrm{N} 1-\mathrm{C} 2-\mathrm{C} 1$ & $113.6(2)$ & $\mathrm{C} 10-\mathrm{C} 9-\mathrm{C} 8$ & $123.05(19)$ \\
\hline $\mathrm{N} 1-\mathrm{C} 2-\mathrm{H} 2 \mathrm{~A}$ & 108.9 & $\mathrm{C} 10-\mathrm{C} 9-\mathrm{N} 3$ & $118.16(18)$ \\
\hline $\mathrm{C} 1-\mathrm{C} 2-\mathrm{H} 2 \mathrm{~A}$ & 108.9 & $\mathrm{C} 8-\mathrm{C} 9-\mathrm{N} 3$ & $118.8(2)$ \\
\hline $\mathrm{N} 1-\mathrm{C} 2-\mathrm{H} 2 \mathrm{~B}$ & 108.9 & $\mathrm{C} 11-\mathrm{C} 10-\mathrm{C} 9$ & $116.52(17)$ \\
\hline $\mathrm{C} 1-\mathrm{C} 2-\mathrm{H} 2 \mathrm{~B}$ & 108.9 & $\mathrm{C} 11-\mathrm{C} 10-\mathrm{H} 10$ & 121.7 \\
\hline $\mathrm{H} 2 \mathrm{~A}-\mathrm{C} 2-\mathrm{H} 2 \mathrm{~B}$ & 107.7 & $\mathrm{C} 9-\mathrm{C} 10-\mathrm{H} 10$ & 121.7 \\
\hline $\mathrm{N} 1-\mathrm{C} 3-\mathrm{C} 4$ & $111.11(18)$ & $\mathrm{C} 10-\mathrm{C} 11-\mathrm{C} 12$ & $122.71(19)$ \\
\hline $\mathrm{N} 1-\mathrm{C} 3-\mathrm{H} 3 \mathrm{~A}$ & 109.4 & $\mathrm{C} 10-\mathrm{C} 11-\mathrm{N} 4$ & $118.60(17)$ \\
\hline $\mathrm{C} 4-\mathrm{C} 3-\mathrm{H} 3 \mathrm{~A}$ & 109.4 & $\mathrm{C} 12-\mathrm{C} 11-\mathrm{N} 4$ & $118.69(18)$ \\
\hline $\mathrm{N} 1-\mathrm{C} 3-\mathrm{H} 3 \mathrm{~B}$ & 109.4 & $\mathrm{C} 7-\mathrm{C} 12-\mathrm{C} 11$ & $119.23(19)$ \\
\hline $\mathrm{C} 4-\mathrm{C} 3-\mathrm{H} 3 \mathrm{~B}$ & 109.4 & $\mathrm{C} 7-\mathrm{C} 12-\mathrm{H} 12$ & 120.4 \\
\hline $\mathrm{H} 3 \mathrm{~A}-\mathrm{C} 3-\mathrm{H} 3 \mathrm{~B}$ & 108 & $\mathrm{C} 11-\mathrm{C} 12-\mathrm{H} 12$ & 120.4 \\
\hline $\mathrm{N} 2-\mathrm{C} 4-\mathrm{C} 3$ & $110.43(17)$ & $\mathrm{O} 1-\mathrm{C} 13-\mathrm{O} 2$ & $126.81(18)$ \\
\hline $\mathrm{N} 2-\mathrm{C} 4-\mathrm{H} 4 \mathrm{~A}$ & 109.6 & $\mathrm{O} 1-\mathrm{C} 13-\mathrm{C} 7$ & $117.20(19)$ \\
\hline $\mathrm{C} 3-\mathrm{C} 4-\mathrm{H} 4 \mathrm{~A}$ & 109.6 & $\mathrm{O} 2-\mathrm{C} 13-\mathrm{C} 7$ & $115.98(18)$ \\
\hline $\mathrm{N} 2-\mathrm{C} 4-\mathrm{H} 4 \mathrm{~B}$ & 109.6 & $\mathrm{C} 3-\mathrm{N} 1-\mathrm{C} 6$ & $109.69(16)$ \\
\hline $\mathrm{C} 3-\mathrm{C} 4-\mathrm{H} 4 \mathrm{~B}$ & 109.6 & $\mathrm{C} 3-\mathrm{N} 1-\mathrm{C} 2$ & $111.54(17)$ \\
\hline $\mathrm{H} 4 \mathrm{~A}-\mathrm{C} 4-\mathrm{H} 4 \mathrm{~B}$ & 108.1 & $\mathrm{C} 6-\mathrm{N} 1-\mathrm{C} 2$ & $108.66(17)$ \\
\hline $\mathrm{N} 2-\mathrm{C} 5-\mathrm{C} 6$ & $110.30(17)$ & $\mathrm{C} 4-\mathrm{N} 2-\mathrm{C} 5$ & $110.20(17)$ \\
\hline $\mathrm{N} 2-\mathrm{C} 5-\mathrm{H} 5 \mathrm{~A}$ & 109.6 & $\mathrm{C} 4-\mathrm{N} 2-\mathrm{H} 21$ & $110(2)$ \\
\hline $\mathrm{C} 6-\mathrm{C} 5-\mathrm{H} 5 \mathrm{~A}$ & 109.6 & $\mathrm{C} 5-\mathrm{N} 2-\mathrm{H} 21$ & $111(2)$ \\
\hline $\mathrm{N} 2-\mathrm{C} 5-\mathrm{H} 5 \mathrm{~B}$ & 109.6 & $\mathrm{C} 4-\mathrm{N} 2-\mathrm{H} 22$ & $108(2)$ \\
\hline $\mathrm{C} 6-\mathrm{C} 5-\mathrm{H} 5 \mathrm{~B}$ & 109.6 & $\mathrm{C} 5-\mathrm{N} 2-\mathrm{H} 22$ & $110.3(19)$ \\
\hline $\mathrm{H} 5 \mathrm{~A}-\mathrm{C} 5-\mathrm{H} 5 \mathrm{~B}$ & 108.1 & $\mathrm{H} 21-\mathrm{N} 2-\mathrm{H} 22$ & $108(3)$ \\
\hline $\mathrm{N} 1-\mathrm{C} 6-\mathrm{C} 5$ & $111.54(18)$ & $\mathrm{O} 3-\mathrm{N} 3-\mathrm{O} 4$ & $124.2(2)$ \\
\hline $\mathrm{N} 1-\mathrm{C} 6-\mathrm{H} 6 \mathrm{~A}$ & 109.3 & $\mathrm{O} 3-\mathrm{N} 3-\mathrm{C} 9$ & $117.8(2)$ \\
\hline $\mathrm{C} 5-\mathrm{C} 6-\mathrm{H} 6 \mathrm{~A}$ & 109.3 & $\mathrm{O} 4-\mathrm{N} 3-\mathrm{C} 9$ & $118.0(2)$ \\
\hline $\mathrm{N} 1-\mathrm{C} 6-\mathrm{H} 6 \mathrm{~B}$ & 109.3 & $\mathrm{O} 6-\mathrm{N} 4-\mathrm{O} 5$ & $123.5(2)$ \\
\hline $\mathrm{C} 5-\mathrm{C} 6-\mathrm{H} 6 \mathrm{~B}$ & 109.3 & $\mathrm{O} 6-\mathrm{N} 4-\mathrm{C} 11$ & 118.33 \\
\hline
\end{tabular}




$\begin{array}{llll}\mathrm{H} 6 \mathrm{~A}-\mathrm{C} 6-\mathrm{H} 6 \mathrm{~B} & 108 & \mathrm{O} 5-\mathrm{N} 4-\mathrm{C} 11 & 118.2(2) \\ \mathrm{N} 1-\mathrm{C} 3-\mathrm{C} 4-\mathrm{N} 2 & -57.7(2) & \mathrm{C} 12-\mathrm{C} 7-\mathrm{C} 13-\mathrm{O} 2 & 15.6(3) \\ \mathrm{N} 2-\mathrm{C} 5-\mathrm{C} 6-\mathrm{N} 1 & 57.5(2) & \mathrm{C} 4-\mathrm{C} 3-\mathrm{N} 1-\mathrm{C} 6 & 58.2(2) \\ \mathrm{C} 12-\mathrm{C} 7-\mathrm{C} 8-\mathrm{C} 9 & -1.2(3) & \mathrm{C} 4-\mathrm{C} 3-\mathrm{N} 1-\mathrm{C} 2 & 178.59(18) \\ \mathrm{C} 13-\mathrm{C} 7-\mathrm{C} 8-\mathrm{C} 9 & 179.11(18) & \mathrm{C} 5-\mathrm{C} 6-\mathrm{N} 1-\mathrm{C} 3 & -58.3(2) \\ \mathrm{C} 7-\mathrm{C} 8-\mathrm{C} 9-\mathrm{C} 10 & 2.1(3) & \mathrm{C} 5-\mathrm{C} 6-\mathrm{N} 1-\mathrm{C} 2 & 179.54(18) \\ \mathrm{C} 7-\mathrm{C} 8-\mathrm{C} 9-\mathrm{N} 3 & -177.62(17) & \mathrm{C} 1-\mathrm{C} 2-\mathrm{N} 1-\mathrm{C} 3 & 65.2(3) \\ \mathrm{C} 8-\mathrm{C} 9-\mathrm{C} 10-\mathrm{C} 11 & -1.2(3) & \mathrm{C} 1-\mathrm{C} 2-\mathrm{N} 1-\mathrm{C} 6 & -173.8(2) \\ \mathrm{N} 3-\mathrm{C} 9-\mathrm{C} 10-\mathrm{C} 11 & 178.58(18) & \mathrm{C} 3-\mathrm{C} 4-\mathrm{N} 2-\mathrm{C} 5 & 56.1(2) \\ \mathrm{C} 9-\mathrm{C} 10-\mathrm{C} 11-\mathrm{C} 12 & -0.6(3) & \mathrm{C} 6-\mathrm{C} 5-\mathrm{N} 2-\mathrm{C} 4 & -55.9(2) \\ \mathrm{C} 9-\mathrm{C} 10-\mathrm{C} 11-\mathrm{N} 4 & 179.50(18) & \mathrm{C} 10-\mathrm{C} 9-\mathrm{N} 3-\mathrm{O} 3 & 165.7(2) \\ \mathrm{C} 8-\mathrm{C} 7-\mathrm{C} 12-\mathrm{C} 11 & -0.5(3) & \mathrm{C} 8-\mathrm{C} 9-\mathrm{N} 3-\mathrm{O} 3 & -14.5(3) \\ \mathrm{C} 13-\mathrm{C} 7-\mathrm{C} 12-\mathrm{C} 11 & 179.18(18) & \mathrm{C} 10-\mathrm{C} 9-\mathrm{N} 3-\mathrm{O} 4 & -15.0(3) \\ \mathrm{C} 10-\mathrm{C} 11-\mathrm{C} 12-\mathrm{C} 7 & 1.5(3) & \mathrm{C} 8-\mathrm{C} 9-\mathrm{N} 3-\mathrm{O} 4 & 164.7(2) \\ \mathrm{N} 4-\mathrm{C} 11-\mathrm{C} 12-\mathrm{C} 7 & -178.69(18) & \mathrm{C} 10-\mathrm{C} 11-\mathrm{N} 4-\mathrm{O} 6 & -174.2(2) \\ \mathrm{C} 8-\mathrm{C} 7-\mathrm{C} 13-\mathrm{O} 1 & 16.0(3) & \mathrm{C} 12-\mathrm{C} 11-\mathrm{N} 4-\mathrm{O} 6 & 5.9(3) \\ \mathrm{C} 12-\mathrm{C} 7-\mathrm{C} 13-\mathrm{O} 1 & -163.64(18) & \mathrm{C} 10-\mathrm{C} 11-\mathrm{N} 4-\mathrm{O} 5 & 6.0(3) \\ \mathrm{C} 8-\mathrm{C} 7-\mathrm{C} 13-\mathrm{O} 2 & -164.73(18) & \mathrm{C} 12-\mathrm{C} 11-\mathrm{N} 4-\mathrm{O} 5 & -173.9(2) \\ & & & \end{array}$

Hydrogen-bond geometry $\left(A,{ }^{\circ}\right)$

\begin{tabular}{lllll}
\hline$D-\mathrm{H} \cdots A$ & $D-\mathrm{H}$ & $\mathrm{H} \cdots A$ & $D \cdots A$ & $D-\mathrm{H} \cdots A$ \\
\hline $\mathrm{N} 2-\mathrm{H} 21 \cdots \mathrm{O} 1$ & $0.91(2)$ & $1.88(2)$ & $2.768(2)$ & $168(3)$ \\
$\mathrm{N} 2-\mathrm{H} 22 \cdots \mathrm{O} 2{ }^{\mathrm{i}}$ & $0.92(2)$ & $1.77(2)$ & $2.684(2)$ & $171(3)$ \\
\hline
\end{tabular}

Symmetry code: (i) $-x,-y,-z$.

4-Methylpiperazin-1-ium 3,5-dinitrobenzoate (II)

Crystal data

$\mathrm{C}_{5} \mathrm{H}_{13} \mathrm{~N}_{2}{ }^{+} \cdot \mathrm{C}_{7} \mathrm{H}_{3} \mathrm{~N}_{2} \mathrm{O}_{6}{ }^{-}$

$M_{r}=312.29$

Triclinic, $P \overline{1}$

Hall symbol: -P 1

$a=7.8023(6) \AA$

$b=10.3920(8) \AA$

$c=10.4770(8) \AA$

$\alpha=73.578(8)^{\circ}$

$\beta=74.289(8)^{\circ}$

$\gamma=71.828(7)^{\circ}$

$V=758.49(11) \AA^{3}$

\section{Data collection}

Oxford Diffraction Xcalibur diffractometer

Graphite monochromator $\omega$ scans

Absorption correction: multi-scan

(CrysAlis RED; Oxford Diffraction, 2009)

$T_{\min }=0.948, T_{\max }=0.952$

4819 measured reflections
$Z=2$

$F(000)=328$

$D_{\mathrm{x}}=1.367 \mathrm{Mg} \mathrm{m}^{-3}$

Mo $K \alpha$ radiation, $\lambda=0.71073 \AA$

Cell parameters from 4819 reflections

$\theta=2.6-25.4^{\circ}$

$\mu=0.11 \mathrm{~mm}^{-1}$

$T=293 \mathrm{~K}$

Prism, orange

$0.48 \times 0.48 \times 0.44 \mathrm{~mm}$

2774 independent reflections

1935 reflections with $I>2 \sigma(I)$

$R_{\text {int }}=0.010$

$\theta_{\text {max }}=25.4^{\circ}, \theta_{\text {min }}=2.6^{\circ}$

$h=-9 \rightarrow 7$

$k=-12 \rightarrow 8$

$l=-12 \rightarrow 12$ 


\section{Refinement}

Refinement on $F^{2}$

Least-squares matrix: full

$R\left[F^{2}>2 \sigma\left(F^{2}\right)\right]=0.044$

$w R\left(F^{2}\right)=0.129$

$S=1.03$

2774 reflections

206 parameters

2 restraints

0 constraints

Primary atom site location: structure-invariant direct methods
Secondary atom site location: difference Fourier map

Hydrogen site location: mixed

$\mathrm{H}$ atoms treated by a mixture of independent and constrained refinement

$w=1 /\left[\sigma^{2}\left(F_{\mathrm{o}}^{2}\right)+(0.0593 P)^{2}+0.1428 P\right]$ where $P=\left(F_{\mathrm{o}}^{2}+2 F_{\mathrm{c}}{ }^{2}\right) / 3$

$(\Delta / \sigma)_{\max }<0.001$

$\Delta \rho_{\max }=0.28{\mathrm{e} \AA^{-3}}^{-3}$

$\Delta \rho_{\min }=-0.15$ e $\AA^{-3}$

Special details

Geometry. All esds (except the esd in the dihedral angle between two 1.s. planes) are estimated using the full covariance matrix. The cell esds are taken into account individually in the estimation of esds in distances, angles and torsion angles; correlations between esds in cell parameters are only used when they are defined by crystal symmetry. An approximate (isotropic) treatment of cell esds is used for estimating esds involving l.s. planes.

Fractional atomic coordinates and isotropic or equivalent isotropic displacement parameters $\left(\AA^{2}\right)$

\begin{tabular}{|c|c|c|c|c|}
\hline & $x$ & $y$ & $z$ & $U_{\text {iso }} * / U_{\text {eq }}$ \\
\hline $\mathrm{O} 1$ & 0.4259 & $-0.25601(14)$ & 0.60539 (19) & $0.1005(6)$ \\
\hline $\mathrm{O} 2$ & $0.3187(2)$ & $-0.28191(15)$ & $0.44153(17)$ & $0.0950(5)$ \\
\hline $\mathrm{O} 3$ & $0.2206(3)$ & $0.1945(2)$ & $0.7260(2)$ & $0.1219(7)$ \\
\hline $\mathrm{O} 4$ & $0.1284(3)$ & $0.37901(16)$ & $0.5805(2)$ & $0.1139(7)$ \\
\hline $\mathrm{O} 5$ & $0.0144(3)$ & $0.3389(2)$ & $0.16451(19)$ & $0.1237(7)$ \\
\hline O6 & $0.0455(3)$ & $0.1363(3)$ & $0.1329(2)$ & $0.1293(8)$ \\
\hline N3 & $0.1771(3)$ & $0.25321(19)$ & $0.6173(2)$ & $0.0806(5)$ \\
\hline N4 & 0.0540 & $0.2122(3)$ & $0.1988(2)$ & $0.0877(6)$ \\
\hline C6 & $0.2667(2)$ & $-0.05607(17)$ & $0.47269(17)$ & $0.0500(4)$ \\
\hline $\mathrm{C} 7$ & $0.2608(2)$ & $0.02602(17)$ & $0.55809(18)$ & 0.0535 \\
\hline $\mathrm{H} 7$ & 0.307549 & -0.013627 & 0.637091 & $0.064 *$ \\
\hline $\mathrm{C} 8$ & $0.1847(2)$ & $0.16762(17)$ & $0.52500(19)$ & $0.0569(4)$ \\
\hline $\mathrm{C} 9$ & $0.1152(2)$ & 0.23142 (19) & $0.4090(2)$ & $0.0625(5)$ \\
\hline $\mathrm{H} 9$ & 0.064143 & 0.326656 & 0.38815 & $0.075^{*}$ \\
\hline $\mathrm{C} 10$ & $0.1249(2)$ & $0.1477(2)$ & $0.32539(19)$ & $0.0606(5)$ \\
\hline $\mathrm{C} 11$ & $0.1974(2)$ & $0.00621(19)$ & $0.35514(18)$ & $0.0568(5)$ \\
\hline H11 & 0.199786 & -0.047271 & 0.29663 & $0.068 *$ \\
\hline $\mathrm{C} 12$ & $0.3453(3)$ & $-0.21207(18)$ & $0.5093(2)$ & $0.0606(5)$ \\
\hline N1 & $0.4346(2)$ & 0.71225 (17) & $0.02379(16)$ & $0.0702(5)$ \\
\hline N2 & $0.4591(3)$ & $0.52702(16)$ & $0.28262(17)$ & $0.0687(5)$ \\
\hline $\mathrm{C} 1$ & $0.3857(4)$ & 0.8479 & $-0.0671(3)$ & $0.1066(9)$ \\
\hline H1A & 0.493157 & 0.865009 & -0.133097 & $0.16^{*}$ \\
\hline H1B & 0.338418 & 0.918742 & -0.015173 & $0.16^{*}$ \\
\hline $\mathrm{H} 1 \mathrm{C}$ & 0.293597 & 0.849066 & -0.112598 & $0.16^{*}$ \\
\hline $\mathrm{C} 2$ & $0.2725(3)$ & $0.6783(2)$ & $0.1169(2)$ & $0.0697(5)$ \\
\hline $\mathrm{H} 2 \mathrm{~A}$ & 0.184008 & 0.681477 & 0.06581 & $0.084 *$ \\
\hline $\mathrm{H} 2 \mathrm{~B}$ & 0.21612 & 0.74668 & 0.172715 & $0.084 *$ \\
\hline $\mathrm{C} 3$ & $0.3197(3)$ & $0.5366(2)$ & $0.2067(2)$ & $0.0739(6)$ \\
\hline
\end{tabular}


supporting information

$\begin{array}{lllll}\text { H3A } & 0.209494 & 0.517861 & 0.270132 & 0.089^{*} \\ \text { H3B } & 0.366896 & 0.467313 & 0.15165 & 0.089^{*} \\ \text { C4 } & 0.6227(3) & 0.5678(2) & 0.1885(2) & 0.0809(6) \\ \text { H4A } & 0.684243 & 0.501297 & 0.131159 & 0.097^{*} \\ \text { H4B } & 0.708662 & 0.568073 & 0.240126 & 0.097^{*} \\ \text { C5 } & 0.5641(3) & 0.7102(2) & 0.1016(2) & 0.0767(6) \\ \text { H5A } & 0.50716 & 0.777105 & 0.15895 & 0.092^{*} \\ \text { H5B } & 0.671413 & 0.736436 & 0.040146 & 0.092^{*} \\ \text { H21 } & 0.412(3) & 0.586(2) & 0.340(2) & 0.092^{*} \\ \text { H22 } & 0.494(3) & 0.4374(18) & 0.329(2) & 0.092^{*}\end{array}$

Atomic displacement parameters $\left(\AA^{2}\right)$

\begin{tabular}{lllllll}
\hline & $U^{11}$ & $U^{22}$ & $U^{33}$ & $U^{12}$ & $U^{13}$ & $U^{23}$ \\
\hline O1 & $0.1400(15)$ & $0.0432(8)$ & $0.1110(13)$ & $0.0019(8)$ & $-0.0587(12)$ & $-0.0017(8)$ \\
O2 & $0.1243(14)$ & $0.0579(9)$ & $0.0982(12)$ & $-0.0057(8)$ & $-0.0139(10)$ & $-0.0373(9)$ \\
O3 & $0.188(2)$ & $0.0895(13)$ & $0.1115(15)$ & $-0.0329(13)$ & $-0.0574(15)$ & $-0.0344(12)$ \\
O4 & $0.1277(14)$ & $0.0523(10)$ & $0.1664(18)$ & $-0.0016(9)$ & $-0.0407(13)$ & $-0.0441(11)$ \\
O5 & $0.1127(14)$ & $0.1017(15)$ & $0.1015(13)$ & $0.0206(11)$ & $-0.0330(11)$ & $0.0208(11)$ \\
O6 & $0.1450(19)$ & $0.149(2)$ & $0.0972(14)$ & $-0.0207(14)$ & $-0.0625(13)$ & $-0.0148(14)$ \\
N3 & $0.0813(12)$ & $0.0582(11)$ & $0.1069(16)$ & $-0.0145(9)$ & $-0.0177(11)$ & $-0.0297(11)$ \\
N4 & $0.0675(12)$ & $0.0988(16)$ & $0.0703(13)$ & $-0.0021(10)$ & $-0.0176(9)$ & $0.0047(12)$ \\
C6 & $0.0438(9)$ & $0.0429(9)$ & $0.0544(10)$ & $-0.0084(7)$ & $-0.0007(7)$ & $-0.0086(8)$ \\
C7 & $0.0489(9)$ & $0.0469(10)$ & $0.0578(10)$ & $-0.0098(7)$ & $-0.0088(8)$ & $-0.0053(8)$ \\
C8 & $0.0514(10)$ & $0.0439(10)$ & $0.0721(12)$ & $-0.0111(7)$ & $-0.0067(9)$ & $-0.0140(9)$ \\
C9 & $0.0484(10)$ & $0.0435(10)$ & $0.0788(13)$ & $-0.0071(7)$ & $-0.0067(9)$ & $0.0018(9)$ \\
C10 & $0.0448(10)$ & $0.0617(12)$ & $0.0590(11)$ & $-0.0073(8)$ & $-0.0074(8)$ & $0.0023(9)$ \\
C11 & $0.0487(10)$ & $0.0597(11)$ & $0.0562(10)$ & $-0.0122(8)$ & $-0.0013(8)$ & $-0.0142(9)$ \\
C12 & $0.0630(11)$ & $0.0420(10)$ & $0.0627(12)$ & $-0.0068(8)$ & $0.0020(9)$ & $-0.0110(9)$ \\
N1 & $0.0768(11)$ & $0.0689(11)$ & $0.0543(9)$ & $-0.0116(8)$ & $-0.0144(8)$ & $-0.0036(8)$ \\
N2 & $0.0975(13)$ & $0.0401(8)$ & $0.0587(10)$ & $0.0023(8)$ & $-0.0235(9)$ & $-0.0102(7)$ \\
C1 & $0.123(2)$ & $0.0942(19)$ & $0.0786(16)$ & $-0.0234(15)$ & $-0.0293(15)$ & $0.0223(14)$ \\
C2 & $0.0695(13)$ & $0.0647(12)$ & $0.0737(13)$ & $-0.0130(10)$ & $-0.0253(10)$ & $-0.0075(10)$ \\
C3 & $0.0870(15)$ & $0.0604(12)$ & $0.0751(13)$ & $-0.0214(10)$ & $-0.0180(11)$ & $-0.0115(10)$ \\
C4 & $0.0717(14)$ & $0.0743(14)$ & $0.0922(16)$ & $0.0063(11)$ & $-0.0325(12)$ & $-0.0237(12)$ \\
C5 & $0.0638(12)$ & $0.0790(14)$ & $0.0780(14)$ & $-0.0189(10)$ & $-0.0099(11)$ & $-0.0061(11)$ \\
& & & & & & \\
\hline & & & & & & \\
\hline
\end{tabular}

Geometric parameters $\left(A,{ }^{o}\right)$

\begin{tabular}{llll}
\hline $\mathrm{O} 1-\mathrm{C} 12$ & $1.233(2)$ & $\mathrm{N} 1-\mathrm{C} 5$ & $1.452(3)$ \\
$\mathrm{O} 2-\mathrm{C} 12$ & $1.241(2)$ & $\mathrm{N} 1-\mathrm{C} 1$ & $1.464(3)$ \\
$\mathrm{O} 3-\mathrm{N} 3$ & $1.213(3)$ & $\mathrm{N} 2-\mathrm{C} 3$ & $1.477(3)$ \\
$\mathrm{O} 4-\mathrm{N} 3$ & $1.219(2)$ & $\mathrm{N} 2-\mathrm{C} 4$ & $1.483(3)$ \\
$\mathrm{O} 5-\mathrm{N} 4$ & $1.224(3)$ & $\mathrm{N} 2-\mathrm{H} 21$ & $0.906(16)$ \\
$\mathrm{O} 6-\mathrm{N} 4$ & $1.212(3)$ & $\mathrm{N} 2-\mathrm{H} 22$ & $0.913(16)$ \\
$\mathrm{N} 3-\mathrm{C} 8$ & $1.467(3)$ & $\mathrm{C} 1-\mathrm{H} 1 \mathrm{~A}$ & 0.96 \\
$\mathrm{~N} 4-\mathrm{C} 10$ & $1.475(3)$ & $\mathrm{C} 1-\mathrm{H} 1 \mathrm{~B}$ & 0.96 \\
$\mathrm{C} 6-\mathrm{C} 7$ & $1.384(2)$ & $\mathrm{C} 1-\mathrm{H} 1 \mathrm{C}$ & 0.96
\end{tabular}




\begin{tabular}{|c|c|c|c|}
\hline $\mathrm{C} 6-\mathrm{C} 11$ & $1.386(2)$ & $\mathrm{C} 2-\mathrm{C} 3$ & $1.502(3)$ \\
\hline $\mathrm{C} 6-\mathrm{C} 12$ & $1.519(2)$ & $\mathrm{C} 2-\mathrm{H} 2 \mathrm{~A}$ & 0.97 \\
\hline $\mathrm{C} 7-\mathrm{C} 8$ & $1.384(2)$ & $\mathrm{C} 2-\mathrm{H} 2 \mathrm{~B}$ & 0.97 \\
\hline $\mathrm{C} 7-\mathrm{H} 7$ & 0.93 & $\mathrm{C} 3-\mathrm{H} 3 \mathrm{~A}$ & 0.97 \\
\hline $\mathrm{C} 8-\mathrm{C} 9$ & $1.374(3)$ & $\mathrm{C} 3-\mathrm{H} 3 \mathrm{~B}$ & 0.97 \\
\hline $\mathrm{C} 9-\mathrm{C} 10$ & $1.373(3)$ & $\mathrm{C} 4-\mathrm{C} 5$ & $1.506(3)$ \\
\hline C9-H9 & 0.93 & $\mathrm{C} 4-\mathrm{H} 4 \mathrm{~A}$ & 0.97 \\
\hline $\mathrm{C} 10-\mathrm{C} 11$ & $1.377(2)$ & $\mathrm{C} 4-\mathrm{H} 4 \mathrm{~B}$ & 0.97 \\
\hline C11-H11 & 0.93 & $\mathrm{C} 5-\mathrm{H} 5 \mathrm{~A}$ & 0.97 \\
\hline $\mathrm{N} 1-\mathrm{C} 2$ & $1.446(2)$ & $\mathrm{C} 5-\mathrm{H} 5 \mathrm{~B}$ & 0.97 \\
\hline $\mathrm{O} 3-\mathrm{N} 3-\mathrm{O} 4$ & $123.6(2)$ & $\mathrm{C} 3-\mathrm{N} 2-\mathrm{H} 22$ & $108.4(15)$ \\
\hline $\mathrm{O} 3-\mathrm{N} 3-\mathrm{C} 8$ & 117.95 & $\mathrm{C} 4-\mathrm{N} 2-\mathrm{H} 22$ & $109.2(14)$ \\
\hline $\mathrm{O} 4-\mathrm{N} 3-\mathrm{C} 8$ & $118.4(2)$ & $\mathrm{H} 21-\mathrm{N} 2-\mathrm{H} 22$ & $111(2)$ \\
\hline $\mathrm{O} 6-\mathrm{N} 4-\mathrm{O} 5$ & $124.2(2)$ & $\mathrm{N} 1-\mathrm{C} 1-\mathrm{H} 1 \mathrm{~A}$ & 109.5 \\
\hline $\mathrm{O} 6-\mathrm{N} 4-\mathrm{C} 10$ & $117.9(2)$ & $\mathrm{N} 1-\mathrm{C} 1-\mathrm{H} 1 \mathrm{~B}$ & 109.5 \\
\hline $\mathrm{O} 5-\mathrm{N} 4-\mathrm{C} 10$ & $117.8(2)$ & $\mathrm{H} 1 \mathrm{~A}-\mathrm{C} 1-\mathrm{H} 1 \mathrm{~B}$ & 109.5 \\
\hline $\mathrm{C} 7-\mathrm{C} 6-\mathrm{C} 11$ & $118.91(16)$ & $\mathrm{N} 1-\mathrm{C} 1-\mathrm{H} 1 \mathrm{C}$ & 109.5 \\
\hline $\mathrm{C} 7-\mathrm{C} 6-\mathrm{C} 12$ & $120.13(16)$ & $\mathrm{H} 1 \mathrm{~A}-\mathrm{C} 1-\mathrm{H} 1 \mathrm{C}$ & 109.5 \\
\hline $\mathrm{C} 11-\mathrm{C} 6-\mathrm{C} 12$ & $120.95(17)$ & $\mathrm{H} 1 \mathrm{~B}-\mathrm{C} 1-\mathrm{H} 1 \mathrm{C}$ & 109.5 \\
\hline $\mathrm{C} 6-\mathrm{C} 7-\mathrm{C} 8$ & $119.47(17)$ & $\mathrm{N} 1-\mathrm{C} 2-\mathrm{C} 3$ & $111.25(17)$ \\
\hline $\mathrm{C} 6-\mathrm{C} 7-\mathrm{H} 7$ & 120.3 & $\mathrm{~N} 1-\mathrm{C} 2-\mathrm{H} 2 \mathrm{~A}$ & 109.4 \\
\hline $\mathrm{C} 8-\mathrm{C} 7-\mathrm{H} 7$ & 120.3 & $\mathrm{C} 3-\mathrm{C} 2-\mathrm{H} 2 \mathrm{~A}$ & 109.4 \\
\hline $\mathrm{C} 9-\mathrm{C} 8-\mathrm{C} 7$ & $122.55(18)$ & $\mathrm{N} 1-\mathrm{C} 2-\mathrm{H} 2 \mathrm{~B}$ & 109.4 \\
\hline $\mathrm{C} 9-\mathrm{C} 8-\mathrm{N} 3$ & $118.53(17)$ & $\mathrm{C} 3-\mathrm{C} 2-\mathrm{H} 2 \mathrm{~B}$ & 109.4 \\
\hline $\mathrm{C} 7-\mathrm{C} 8-\mathrm{N} 3$ & $118.92(18)$ & $\mathrm{H} 2 \mathrm{~A}-\mathrm{C} 2-\mathrm{H} 2 \mathrm{~B}$ & 108 \\
\hline $\mathrm{C} 10-\mathrm{C} 9-\mathrm{C} 8$ & $116.73(16)$ & $\mathrm{N} 2-\mathrm{C} 3-\mathrm{C} 2$ & $110.67(16)$ \\
\hline $\mathrm{C} 10-\mathrm{C} 9-\mathrm{H} 9$ & 121.6 & $\mathrm{~N} 2-\mathrm{C} 3-\mathrm{H} 3 \mathrm{~A}$ & 109.5 \\
\hline $\mathrm{C} 8-\mathrm{C} 9-\mathrm{H} 9$ & 121.6 & $\mathrm{C} 2-\mathrm{C} 3-\mathrm{H} 3 \mathrm{~A}$ & 109.5 \\
\hline $\mathrm{C} 9-\mathrm{C} 10-\mathrm{C} 11$ & $122.66(18)$ & $\mathrm{N} 2-\mathrm{C} 3-\mathrm{H} 3 \mathrm{~B}$ & 109.5 \\
\hline $\mathrm{C} 9-\mathrm{C} 10-\mathrm{N} 4$ & $118.52(19)$ & $\mathrm{C} 2-\mathrm{C} 3-\mathrm{H} 3 \mathrm{~B}$ & 109.5 \\
\hline $\mathrm{C} 11-\mathrm{C} 10-\mathrm{N} 4$ & $118.8(2)$ & $\mathrm{H} 3 \mathrm{~A}-\mathrm{C} 3-\mathrm{H} 3 \mathrm{~B}$ & 108.1 \\
\hline $\mathrm{C} 10-\mathrm{C} 11-\mathrm{C} 6$ & $119.67(18)$ & $\mathrm{N} 2-\mathrm{C} 4-\mathrm{C} 5$ & $109.78(16)$ \\
\hline $\mathrm{C} 10-\mathrm{C} 11-\mathrm{H} 11$ & 120.2 & $\mathrm{~N} 2-\mathrm{C} 4-\mathrm{H} 4 \mathrm{~A}$ & 109.7 \\
\hline $\mathrm{C} 6-\mathrm{C} 11-\mathrm{H} 11$ & 120.2 & $\mathrm{C} 5-\mathrm{C} 4-\mathrm{H} 4 \mathrm{~A}$ & 109.7 \\
\hline $\mathrm{O} 1-\mathrm{C} 12-\mathrm{O} 2$ & $126.92(18)$ & $\mathrm{N} 2-\mathrm{C} 4-\mathrm{H} 4 \mathrm{~B}$ & 109.7 \\
\hline $\mathrm{O} 1-\mathrm{C} 12-\mathrm{C} 6$ & $116.25(18)$ & $\mathrm{C} 5-\mathrm{C} 4-\mathrm{H} 4 \mathrm{~B}$ & 109.7 \\
\hline $\mathrm{O} 2-\mathrm{C} 12-\mathrm{C} 6$ & $116.81(19)$ & $\mathrm{H} 4 \mathrm{~A}-\mathrm{C} 4-\mathrm{H} 4 \mathrm{~B}$ & 108.2 \\
\hline $\mathrm{C} 2-\mathrm{N} 1-\mathrm{C} 5$ & $108.81(15)$ & $\mathrm{N} 1-\mathrm{C} 5-\mathrm{C} 4$ & $110.61(18)$ \\
\hline $\mathrm{C} 2-\mathrm{N} 1-\mathrm{C} 1$ & $110.74(18)$ & $\mathrm{N} 1-\mathrm{C} 5-\mathrm{H} 5 \mathrm{~A}$ & 109.5 \\
\hline $\mathrm{C} 5-\mathrm{N} 1-\mathrm{C} 1$ & $110.91(18)$ & $\mathrm{C} 4-\mathrm{C} 5-\mathrm{H} 5 \mathrm{~A}$ & 109.5 \\
\hline $\mathrm{C} 3-\mathrm{N} 2-\mathrm{C} 4$ & $110.66(16)$ & $\mathrm{N} 1-\mathrm{C} 5-\mathrm{H} 5 \mathrm{~B}$ & 109.5 \\
\hline $\mathrm{C} 3-\mathrm{N} 2-\mathrm{H} 21$ & $110.0(15)$ & $\mathrm{C} 4-\mathrm{C} 5-\mathrm{H} 5 \mathrm{~B}$ & 109.5 \\
\hline $\mathrm{C} 4-\mathrm{N} 2-\mathrm{H} 21$ & $107.1(14)$ & $\mathrm{H} 5 \mathrm{~A}-\mathrm{C} 5-\mathrm{H} 5 \mathrm{~B}$ & 108.1 \\
\hline $\mathrm{C} 11-\mathrm{C} 6-\mathrm{C} 7-\mathrm{C} 8$ & $0.5(2)$ & $\mathrm{C} 9-\mathrm{C} 10-\mathrm{C} 11-\mathrm{C} 6$ & $-1.0(3)$ \\
\hline $\mathrm{C} 12-\mathrm{C} 6-\mathrm{C} 7-\mathrm{C} 8$ & $-178.42(15)$ & $\mathrm{N} 4-\mathrm{C} 10-\mathrm{C} 11-\mathrm{C} 6$ & $179.18(15)$ \\
\hline $\mathrm{C} 6-\mathrm{C} 7-\mathrm{C} 8-\mathrm{C} 9$ & $-0.6(3)$ & $\mathrm{C} 7-\mathrm{C} 6-\mathrm{C} 11-\mathrm{C} 10$ & $0.2(2)$ \\
\hline
\end{tabular}




\begin{tabular}{llll}
$\mathrm{C} 6-\mathrm{C} 7-\mathrm{C} 8-\mathrm{N} 3$ & $179.23(15)$ & $\mathrm{C} 12-\mathrm{C} 6-\mathrm{C} 11-\mathrm{C} 10$ & $179.17(15)$ \\
$\mathrm{O} 3-\mathrm{N} 3-\mathrm{C} 8-\mathrm{C} 9$ & $172.3(2)$ & $\mathrm{C} 7-\mathrm{C} 6-\mathrm{C} 12-\mathrm{O} 1$ & $-10.5(2)$ \\
$\mathrm{O} 4-\mathrm{N} 3-\mathrm{C} 8-\mathrm{C} 9$ & $-8.0(3)$ & $\mathrm{C} 11-\mathrm{C} 6-\mathrm{C} 12-\mathrm{O} 1$ & $170.56(17)$ \\
$\mathrm{O} 3-\mathrm{N} 3-\mathrm{C} 8-\mathrm{C} 7$ & $-7.5(3)$ & $\mathrm{C} 7-\mathrm{C} 6-\mathrm{C} 12-\mathrm{O} 2$ & $168.17(16)$ \\
$\mathrm{O} 4-\mathrm{N} 3-\mathrm{C} 8-\mathrm{C} 7$ & $172.18(18)$ & $\mathrm{C} 11-\mathrm{C} 6-\mathrm{C} 12-\mathrm{O} 2$ & $-10.8(2)$ \\
$\mathrm{C} 7-\mathrm{C} 8-\mathrm{C} 9-\mathrm{C} 10$ & $-0.2(3)$ & $\mathrm{C} 5-\mathrm{N} 1-\mathrm{C} 2-\mathrm{C} 3$ & $-60.3(2)$ \\
$\mathrm{N} 3-\mathrm{C} 8-\mathrm{C} 9-\mathrm{C} 10$ & $-179.99(15)$ & $\mathrm{C} 1-\mathrm{N} 1-\mathrm{C} 2-\mathrm{C} 3$ & $177.52(19)$ \\
$\mathrm{C} 8-\mathrm{C} 9-\mathrm{C} 10-\mathrm{C} 11$ & $1.0(3)$ & $\mathrm{C} 4-\mathrm{N} 2-\mathrm{C} 3-\mathrm{C} 2$ & $-54.0(2)$ \\
$\mathrm{C} 8-\mathrm{C} 9-\mathrm{C} 10-\mathrm{N} 4$ & $-179.21(15)$ & $\mathrm{N} 1-\mathrm{C} 2-\mathrm{C} 3-\mathrm{N} 2$ & $57.2(2)$ \\
$\mathrm{O} 6-\mathrm{N} 4-\mathrm{C} 10-\mathrm{C} 9$ & $-172.5(2)$ & $\mathrm{C} 3-\mathrm{N} 2-\mathrm{C} 4-\mathrm{C} 5$ & $54.9(2)$ \\
$\mathrm{O} 5-\mathrm{N} 4-\mathrm{C} 10-\mathrm{C} 9$ & $10.3(3)$ & $\mathrm{C} 2-\mathrm{N} 1-\mathrm{C} 5-\mathrm{C} 4$ & $61.4(2)$ \\
$\mathrm{O} 6-\mathrm{N} 4-\mathrm{C} 10-\mathrm{C} 11$ & $7.3(3)$ & $\mathrm{C} 1-\mathrm{N} 1-\mathrm{C} 5-\mathrm{C} 4$ & $-176.53(19)$ \\
$\mathrm{O} 5-\mathrm{N} 4-\mathrm{C} 10-\mathrm{C} 11$ & $-169.91(18)$ & $\mathrm{N} 2-\mathrm{C} 4-\mathrm{C} 5-\mathrm{N} 1$ & $-59.1(2)$ \\
\hline
\end{tabular}

Hydrogen-bond geometry $\left(\AA,{ }^{\circ}\right)$

\begin{tabular}{lllll}
\hline$D-\mathrm{H} \cdots A$ & $D-\mathrm{H}$ & $\mathrm{H} \cdots A$ & $D \cdots A$ & $D-\mathrm{H} \cdots A$ \\
\hline $\mathrm{N} 2-\mathrm{H} 21 \cdots \mathrm{O} 2^{\mathrm{i}}$ & $0.91(2)$ & $1.82(2)$ & $2.728(2)$ & $175(2)$ \\
$\mathrm{N} 2-\mathrm{H} 22 \cdots \mathrm{O} 1^{\mathrm{ii}}$ & $0.91(2)$ & $1.78(2)$ & $2.691(2)$ & $172(2)$ \\
\hline
\end{tabular}

Symmetry codes: (i) $x, y+1, z$; (ii) $-x+1,-y,-z+1$.

4-Methylpiperazin-1-ium 4-iodobenzoate (III)

Crystal data

$\mathrm{C}_{5} \mathrm{H}_{13} \mathrm{~N}_{2}{ }^{+} \cdot \mathrm{C}_{7} \mathrm{H}_{4} \mathrm{IO}_{2}$

$M_{r}=348.17$

Triclinic, $P \overline{1}$

Hall symbol: -P 1

$a=6.2418$ (4) $\AA$

$b=9.5465(8) \AA$

$c=12.5346(9) \AA$

$\alpha=110.708(8)^{\circ}$

$\beta=90.235(5)^{\circ}$

$\gamma=101.559(6)^{\circ}$

$V=682.19(9) \AA^{3}$

\section{Data collection}

Oxford Diffraction Xcalibur diffractometer

Graphite monochromator

$\omega$ scans

Absorption correction: multi-scan

(CrysAlis RED; Oxford Diffraction, 2009)

$T_{\text {min }}=0.515, T_{\text {max }}=0.626$

4189 measured reflections

\section{Refinement}

Refinement on $F^{2}$

Least-squares matrix: full

$R\left[F^{2}>2 \sigma\left(F^{2}\right)\right]=0.020$

$w R\left(F^{2}\right)=0.051$

$S=1.11$
$Z=2$

$F(000)=344$

$D_{\mathrm{x}}=1.695 \mathrm{Mg} \mathrm{m}^{-3}$

Mo $K \alpha$ radiation, $\lambda=0.71073 \AA$

Cell parameters from 4189 reflections

$\theta=3.3-25.4^{\circ}$

$\mu=2.34 \mathrm{~mm}^{-1}$

$T=293 \mathrm{~K}$

Rods, colourless

$0.48 \times 0.24 \times 0.2 \mathrm{~mm}$

2492 independent reflections

2324 reflections with $I>2 \sigma(I)$

$R_{\text {int }}=0.011$

$\theta_{\max }=25.4^{\circ}, \theta_{\min }=3.3^{\circ}$

$h=-7 \rightarrow 7$

$k=-11 \rightarrow 11$

$l=-15 \rightarrow 14$

2492 reflections

160 parameters

2 restraints

0 constraints 
Primary atom site location: structure-invariant direct methods

Secondary atom site location: difference Fourier map

Hydrogen site location: mixed
$\mathrm{H}$ atoms treated by a mixture of independent and constrained refinement

$w=1 /\left[\sigma^{2}\left(F_{\mathrm{o}}^{2}\right)+(0.0289 P)^{2}+0.2845 P\right]$ where $P=\left(F_{\mathrm{o}}{ }^{2}+2 F_{\mathrm{c}}{ }^{2}\right) / 3$

$(\Delta / \sigma)_{\max }<0.001$

$\Delta \rho_{\max }=0.37$ e $\AA^{-3}$

$\Delta \rho_{\min }=-0.95$ e $\AA^{-3}$

Special details

Geometry. All esds (except the esd in the dihedral angle between two 1.s. planes) are estimated using the full covariance matrix. The cell esds are taken into account individually in the estimation of esds in distances, angles and torsion angles; correlations between esds in cell parameters are only used when they are defined by crystal symmetry. An approximate (isotropic) treatment of cell esds is used for estimating esds involving l.s. planes.

Fractional atomic coordinates and isotropic or equivalent isotropic displacement parameters $\left(\AA^{2}\right)$

\begin{tabular}{lllll}
\hline & $x$ & $y$ & $z$ & $U_{\text {iso }} / U_{\text {eq }}$ \\
\hline C1 & $1.2514(5)$ & $0.7762(5)$ & $0.1396(3)$ & $0.0712(11)$ \\
H1A & 1.242455 & 0.690853 & 0.068934 & $0.107^{*}$ \\
H1B & 1.283901 & 0.870031 & 0.124947 & $0.107^{*}$ \\
H1C & 1.365551 & 0.776131 & 0.19115 & $0.107^{*}$ \\
C2 & $1.0513(4)$ & $0.8911(3)$ & $0.2981(2)$ & $0.0429(6)$ \\
H2A & 1.085517 & 0.986567 & 0.284191 & $0.051^{*}$ \\
H2B & 1.167221 & 0.892502 & 0.350502 & $0.051^{*}$ \\
C3 & $0.8371(4)$ & $0.8786(3)$ & $0.3512(2)$ & $0.0428(6)$ \\
H3A & 0.847548 & 0.963711 & 0.423379 & $0.051^{*}$ \\
H3B & 0.722435 & 0.883465 & 0.301016 & $0.051^{*}$ \\
C4 & $0.7745(4)$ & $0.6017(3)$ & $0.2629(2)$ & $0.0432(6)$ \\
H4A & 0.656473 & 0.596833 & 0.210215 & $0.052^{*}$ \\
H4B & 0.745692 & 0.506984 & 0.277895 & $0.052^{*}$ \\
C5 & $0.9887(4)$ & $0.6188(3)$ & $0.2099(2)$ & $0.0424(6)$ \\
H5A & 1.10447 & 0.614721 & 0.259906 & $0.051^{*}$ \\
H5B & 0.980005 & 0.534325 & 0.137531 & $0.051^{*}$ \\
C6 & $0.6076(4)$ & $0.2567(3)$ & $0.3636(2)$ & $0.0296(5)$ \\
C7 & $0.6850(4)$ & $0.1909(3)$ & $0.2577(2)$ & $0.0339(5)$ \\
H7 & 0.812059 & 0.153084 & 0.253927 & $0.041^{*}$ \\
C8 & $0.5770(4)$ & $0.1804(3)$ & $0.1574(2)$ & $0.0355(5)$ \\
H8 & 0.629598 & 0.134593 & 0.086951 & $0.043^{*}$ \\
C9 & $0.3881(4)$ & $0.2396(3)$ & $0.1635(2)$ & $0.0312(5)$ \\
C10 & $0.3100(4)$ & $0.3057(3)$ & $0.2684(2)$ & $0.0332(5)$ \\
H10 & 0.184232 & 0.345025 & 0.272511 & $0.04^{*}$ \\
C11 & $0.4189(4)$ & $0.3135(3)$ & $0.3679(2)$ & $0.0336(5)$ \\
H11 & 0.364548 & 0.357276 & 0.438151 & $0.04^{*}$ \\
C12 & $0.7285(4)$ & $0.2674(3)$ & $0.4722(2)$ & $0.0345(5)$ \\
I1 & $0.22668(3)$ & $0.23210(2)$ & $0.01333(2)$ & $0.03947(7)$ \\
N1 & $1.0417(3)$ & $0.7631(3)$ & $0.19094(18)$ & $0.0384(5)$ \\
N2 & $0.7815(3)$ & $0.7327(3)$ & $0.37083(19)$ & $0.0393(5)$ \\
O1 & $0.6295(3)$ & $0.2895(3)$ & $0.56080(16)$ & $0.0567(6)$ \\
O2 & $0.9237(3)$ & $0.2528(3)$ & $0.46569(16)$ & $0.0467(5)$ \\
& & & &
\end{tabular}


supporting information

\begin{tabular}{lllll}
$\mathrm{H} 21$ & $0.876(4)$ & $0.728(4)$ & $0.420(2)$ & $0.056^{*}$ \\
$\mathrm{H} 22$ & $0.653(4)$ & $0.728(4)$ & $0.400(3)$ & $0.056^{*}$ \\
\hline
\end{tabular}

Atomic displacement parameters $\left(A^{2}\right)$

\begin{tabular}{lllllll}
\hline & $U^{11}$ & $U^{22}$ & $U^{33}$ & $U^{12}$ & $U^{13}$ & $U^{23}$ \\
\hline C1 & $0.0331(15)$ & $0.135(3)$ & $0.056(2)$ & $0.0112(18)$ & $0.0100(14)$ & $0.051(2)$ \\
C2 & $0.0390(14)$ & $0.0381(14)$ & $0.0506(16)$ & $-0.0024(11)$ & $-0.0094(12)$ & $0.0208(12)$ \\
C3 & $0.0459(14)$ & $0.0405(14)$ & $0.0395(14)$ & $0.0171(12)$ & $-0.0055(11)$ & $0.0075(12)$ \\
C4 & $0.0434(14)$ & $0.0365(14)$ & $0.0484(16)$ & $-0.0033(11)$ & $-0.0035(12)$ & $0.0204(12)$ \\
C5 & $0.0455(15)$ & $0.0428(14)$ & $0.0373(14)$ & $0.0159(12)$ & $0.0009(11)$ & $0.0092(12)$ \\
C6 & $0.0241(10)$ & $0.0347(12)$ & $0.0346(12)$ & $0.0059(9)$ & $0.0019(9)$ & $0.0183(10)$ \\
C7 & $0.0272(11)$ & $0.0423(13)$ & $0.0378(13)$ & $0.0142(10)$ & $0.0040(9)$ & $0.0174(11)$ \\
C8 & $0.0362(13)$ & $0.0404(13)$ & $0.0311(12)$ & $0.0142(10)$ & $0.0056(10)$ & $0.0110(11)$ \\
C9 & $0.0291(11)$ & $0.0343(12)$ & $0.0326(12)$ & $0.0050(9)$ & $-0.0031(9)$ & $0.0162(10)$ \\
C10 & $0.0239(11)$ & $0.0430(13)$ & $0.0380(13)$ & $0.0108(10)$ & $0.0029(9)$ & $0.0193(11)$ \\
C11 & $0.0299(11)$ & $0.0446(14)$ & $0.0321(12)$ & $0.0117(10)$ & $0.0066(9)$ & $0.0186(11)$ \\
C12 & $0.0258(11)$ & $0.0471(14)$ & $0.0354(13)$ & $0.0087(10)$ & $0.0017(9)$ & $0.0203(11)$ \\
I1 & $0.03737(10)$ & $0.05212(12)$ & $0.03188(10)$ & $0.01315(7)$ & $-0.00148(7)$ & $0.01691(8)$ \\
N1 & $0.0269(10)$ & $0.0595(14)$ & $0.0346(11)$ & $0.0059(9)$ & $0.0023(8)$ & $0.0260(10)$ \\
N2 & $0.0279(10)$ & $0.0639(14)$ & $0.0327(11)$ & $0.0124(10)$ & $0.0036(8)$ & $0.0241(11)$ \\
O1 & $0.0371(10)$ & $0.1100(18)$ & $0.0370(11)$ & $0.0261(11)$ & $0.0097(8)$ & $0.0379(12)$ \\
O2 & $0.0319(9)$ & $0.0780(14)$ & $0.0400(10)$ & $0.0216(9)$ & $0.0035(8)$ & $0.0278(10)$ \\
& & & & & & \\
\hline
\end{tabular}

Geometric parameters $\left(A,{ }^{\circ}\right)$

\begin{tabular}{llll}
\hline $\mathrm{C} 1-\mathrm{N} 1$ & $1.464(3)$ & $\mathrm{C} 5-\mathrm{H} 5 \mathrm{~B}$ & 0.97 \\
$\mathrm{C} 1-\mathrm{H} 1 \mathrm{~A}$ & 0.96 & $\mathrm{C} 6-\mathrm{C} 11$ & $1.386(3)$ \\
$\mathrm{C} 1-\mathrm{H} 1 \mathrm{~B}$ & 0.96 & $\mathrm{C} 6-\mathrm{C} 7$ & $1.388(3)$ \\
$\mathrm{C} 1-\mathrm{H} 1 \mathrm{C}$ & 0.96 & $\mathrm{C} 6-\mathrm{C} 12$ & $1.514(3)$ \\
$\mathrm{C} 2-\mathrm{N} 1$ & $1.453(4)$ & $\mathrm{C} 7-\mathrm{C} 8$ & $1.386(4)$ \\
$\mathrm{C} 2-\mathrm{C} 3$ & $1.498(4)$ & $\mathrm{C} 7-\mathrm{H} 7$ & 0.93 \\
$\mathrm{C} 2-\mathrm{H} 2 \mathrm{~A}$ & 0.97 & $\mathrm{C} 8-\mathrm{C} 9$ & $1.398(3)$ \\
$\mathrm{C} 2-\mathrm{H} 2 \mathrm{~B}$ & 0.97 & $\mathrm{C} 8-\mathrm{H} 8$ & 0.93 \\
$\mathrm{C} 3-\mathrm{N} 2$ & $1.474(4)$ & $\mathrm{C} 9-\mathrm{C} 10$ & $1.381(3)$ \\
$\mathrm{C} 3-\mathrm{H} 3 \mathrm{~A}$ & 0.97 & $\mathrm{C} 9-\mathrm{I} 1$ & $2.103(2)$ \\
$\mathrm{C} 3-\mathrm{H} 3 \mathrm{~B}$ & 0.97 & $\mathrm{C} 10-\mathrm{C} 11$ & $1.389(3)$ \\
$\mathrm{C} 4-\mathrm{N} 2$ & $1.475(4)$ & $\mathrm{C} 10-\mathrm{H} 10$ & 0.93 \\
$\mathrm{C} 4-\mathrm{C} 5$ & $1.501(4)$ & $\mathrm{C} 11-\mathrm{H} 11$ & 0.93 \\
$\mathrm{C} 4-\mathrm{H} 4 \mathrm{~A}$ & 0.97 & $\mathrm{C} 12-\mathrm{O} 1$ & $1.246(3)$ \\
$\mathrm{C} 4-\mathrm{H} 4 \mathrm{~B}$ & 0.97 & $\mathrm{C} 12-\mathrm{O} 2$ & $1.254(3)$ \\
$\mathrm{C} 5-\mathrm{N} 1$ & $\mathrm{~N} 2-\mathrm{H} 21$ & $0.874(18)$ \\
$\mathrm{C} 5-\mathrm{H} 5 \mathrm{~A}$ & $1.454(4)$ & $\mathrm{N} 2-\mathrm{H} 22$ & $0.881(18)$ \\
& 0.97 & $\mathrm{C} 11-\mathrm{C} 6-\mathrm{C} 7$ & $118.6(2)$ \\
$\mathrm{N} 1-\mathrm{C} 1-\mathrm{H} 1 \mathrm{~A}$ & & $\mathrm{C} 11-\mathrm{C} 6-\mathrm{C} 12$ & $120.8(2)$ \\
$\mathrm{N} 1-\mathrm{C} 1-\mathrm{H} 1 \mathrm{~B}$ & 109.5 & $\mathrm{C} 7-\mathrm{C} 6-\mathrm{C} 12$ & $120.6(2)$ \\
$\mathrm{H} 1 \mathrm{~A}-\mathrm{C} 1-\mathrm{H} 1 \mathrm{~B}$ & 109.5 & $\mathrm{C} 8-\mathrm{C} 7-\mathrm{C} 6$ & $121.5(2)$ \\
$\mathrm{N} 1-\mathrm{C} 1-\mathrm{H} 1 \mathrm{C}$ & 109.5 &
\end{tabular}




\begin{tabular}{|c|c|c|c|}
\hline $\mathrm{H} 1 \mathrm{~A}-\mathrm{C} 1-\mathrm{H} 1 \mathrm{C}$ & 109.5 & $\mathrm{C} 8-\mathrm{C} 7-\mathrm{H} 7$ & 119.3 \\
\hline $\mathrm{H} 1 \mathrm{~B}-\mathrm{C} 1-\mathrm{H} 1 \mathrm{C}$ & 109.5 & $\mathrm{C} 6-\mathrm{C} 7-\mathrm{H} 7$ & 119.3 \\
\hline $\mathrm{N} 1-\mathrm{C} 2-\mathrm{C} 3$ & $110.8(2)$ & $\mathrm{C} 7-\mathrm{C} 8-\mathrm{C} 9$ & $119.1(2)$ \\
\hline $\mathrm{N} 1-\mathrm{C} 2-\mathrm{H} 2 \mathrm{~A}$ & 109.5 & $\mathrm{C} 7-\mathrm{C} 8-\mathrm{H} 8$ & 120.4 \\
\hline $\mathrm{C} 3-\mathrm{C} 2-\mathrm{H} 2 \mathrm{~A}$ & 109.5 & $\mathrm{C} 9-\mathrm{C} 8-\mathrm{H} 8$ & 120.4 \\
\hline $\mathrm{N} 1-\mathrm{C} 2-\mathrm{H} 2 \mathrm{~B}$ & 109.5 & $\mathrm{C} 10-\mathrm{C} 9-\mathrm{C} 8$ & $119.9(2)$ \\
\hline $\mathrm{C} 3-\mathrm{C} 2-\mathrm{H} 2 \mathrm{~B}$ & 109.5 & $\mathrm{C} 10-\mathrm{C} 9-\mathrm{I} 1$ & $120.07(17)$ \\
\hline $\mathrm{H} 2 \mathrm{~A}-\mathrm{C} 2-\mathrm{H} 2 \mathrm{~B}$ & 108.1 & $\mathrm{C} 8-\mathrm{C} 9-\mathrm{I} 1$ & $120.00(18)$ \\
\hline $\mathrm{N} 2-\mathrm{C} 3-\mathrm{C} 2$ & $110.0(2)$ & $\mathrm{C} 9-\mathrm{C} 10-\mathrm{C} 11$ & $120.1(2)$ \\
\hline $\mathrm{N} 2-\mathrm{C} 3-\mathrm{H} 3 \mathrm{~A}$ & 109.7 & $\mathrm{C} 9-\mathrm{C} 10-\mathrm{H} 10$ & 119.9 \\
\hline $\mathrm{C} 2-\mathrm{C} 3-\mathrm{H} 3 \mathrm{~A}$ & 109.7 & $\mathrm{C} 11-\mathrm{C} 10-\mathrm{H} 10$ & 119.9 \\
\hline $\mathrm{N} 2-\mathrm{C} 3-\mathrm{H} 3 \mathrm{~B}$ & 109.7 & $\mathrm{C} 6-\mathrm{C} 11-\mathrm{C} 10$ & $120.8(2)$ \\
\hline $\mathrm{C} 2-\mathrm{C} 3-\mathrm{H} 3 \mathrm{~B}$ & 109.7 & $\mathrm{C} 6-\mathrm{C} 11-\mathrm{H} 11$ & 119.6 \\
\hline $\mathrm{H} 3 \mathrm{~A}-\mathrm{C} 3-\mathrm{H} 3 \mathrm{~B}$ & 108.2 & $\mathrm{C} 10-\mathrm{C} 11-\mathrm{H} 11$ & 119.6 \\
\hline $\mathrm{N} 2-\mathrm{C} 4-\mathrm{C} 5$ & $110.2(2)$ & $\mathrm{O} 1-\mathrm{C} 12-\mathrm{O} 2$ & $124.5(2)$ \\
\hline $\mathrm{N} 2-\mathrm{C} 4-\mathrm{H} 4 \mathrm{~A}$ & 109.6 & $\mathrm{O} 1-\mathrm{C} 12-\mathrm{C} 6$ & $118.7(2)$ \\
\hline $\mathrm{C} 5-\mathrm{C} 4-\mathrm{H} 4 \mathrm{~A}$ & 109.6 & $\mathrm{O} 2-\mathrm{C} 12-\mathrm{C} 6$ & $116.7(2)$ \\
\hline $\mathrm{N} 2-\mathrm{C} 4-\mathrm{H} 4 \mathrm{~B}$ & 109.6 & $\mathrm{C} 2-\mathrm{N} 1-\mathrm{C} 5$ & $110.3(2)$ \\
\hline $\mathrm{C} 5-\mathrm{C} 4-\mathrm{H} 4 \mathrm{~B}$ & 109.6 & $\mathrm{C} 2-\mathrm{N} 1-\mathrm{C} 1$ & $110.5(2)$ \\
\hline $\mathrm{H} 4 \mathrm{~A}-\mathrm{C} 4-\mathrm{H} 4 \mathrm{~B}$ & 108.1 & $\mathrm{C} 5-\mathrm{N} 1-\mathrm{C} 1$ & $109.6(2)$ \\
\hline $\mathrm{N} 1-\mathrm{C} 5-\mathrm{C} 4$ & $111.1(2)$ & $\mathrm{C} 3-\mathrm{N} 2-\mathrm{C} 4$ & $110.6(2)$ \\
\hline $\mathrm{N} 1-\mathrm{C} 5-\mathrm{H} 5 \mathrm{~A}$ & 109.4 & $\mathrm{C} 3-\mathrm{N} 2-\mathrm{H} 21$ & $112(2)$ \\
\hline $\mathrm{C} 4-\mathrm{C} 5-\mathrm{H} 5 \mathrm{~A}$ & 109.4 & $\mathrm{C} 4-\mathrm{N} 2-\mathrm{H} 21$ & $108(2)$ \\
\hline $\mathrm{N} 1-\mathrm{C} 5-\mathrm{H} 5 \mathrm{~B}$ & 109.4 & $\mathrm{C} 3-\mathrm{N} 2-\mathrm{H} 22$ & $108(2)$ \\
\hline $\mathrm{C} 4-\mathrm{C} 5-\mathrm{H} 5 \mathrm{~B}$ & 109.4 & $\mathrm{C} 4-\mathrm{N} 2-\mathrm{H} 22$ & $111(2)$ \\
\hline $\mathrm{H} 5 \mathrm{~A}-\mathrm{C} 5-\mathrm{H} 5 \mathrm{~B}$ & 108 & $\mathrm{H} 21-\mathrm{N} 2-\mathrm{H} 22$ & $108(3)$ \\
\hline $\mathrm{N} 1-\mathrm{C} 2-\mathrm{C} 3-\mathrm{N} 2$ & $58.1(3)$ & $\mathrm{C} 9-\mathrm{C} 10-\mathrm{C} 11-\mathrm{C} 6$ & $0.6(4)$ \\
\hline $\mathrm{N} 2-\mathrm{C} 4-\mathrm{C} 5-\mathrm{N} 1$ & $-56.7(3)$ & $\mathrm{C} 11-\mathrm{C} 6-\mathrm{C} 12-\mathrm{O} 1$ & $18.7(4)$ \\
\hline $\mathrm{C} 11-\mathrm{C} 6-\mathrm{C} 7-\mathrm{C} 8$ & $-0.4(4)$ & $\mathrm{C} 7-\mathrm{C} 6-\mathrm{C} 12-\mathrm{O} 1$ & $-161.8(2)$ \\
\hline $\mathrm{C} 12-\mathrm{C} 6-\mathrm{C} 7-\mathrm{C} 8$ & $-179.8(2)$ & $\mathrm{C} 11-\mathrm{C} 6-\mathrm{C} 12-\mathrm{O} 2$ & $-161.4(2)$ \\
\hline $\mathrm{C} 6-\mathrm{C} 7-\mathrm{C} 8-\mathrm{C} 9$ & $1.0(4)$ & $\mathrm{C} 7-\mathrm{C} 6-\mathrm{C} 12-\mathrm{O} 2$ & $18.0(4)$ \\
\hline $\mathrm{C} 7-\mathrm{C} 8-\mathrm{C} 9-\mathrm{C} 10$ & $-0.8(4)$ & $\mathrm{C} 3-\mathrm{C} 2-\mathrm{N} 1-\mathrm{C} 5$ & $-58.7(3)$ \\
\hline $\mathrm{C} 7-\mathrm{C} 8-\mathrm{C} 9-\mathrm{I} 1$ & $177.94(18)$ & $\mathrm{C} 3-\mathrm{C} 2-\mathrm{N} 1-\mathrm{C} 1$ & $180.0(2)$ \\
\hline $\mathrm{C} 8-\mathrm{C} 9-\mathrm{C} 10-\mathrm{C} 11$ & $0.0(4)$ & $\mathrm{C} 4-\mathrm{C} 5-\mathrm{N} 1-\mathrm{C} 2$ & $58.0(3)$ \\
\hline $\mathrm{I} 1-\mathrm{C} 9-\mathrm{C} 10-\mathrm{C} 11$ & $-178.72(18)$ & $\mathrm{C} 4-\mathrm{C} 5-\mathrm{N} 1-\mathrm{C} 1$ & $179.9(2)$ \\
\hline $\mathrm{C} 7-\mathrm{C} 6-\mathrm{C} 11-\mathrm{C} 10$ & $-0.4(4)$ & $\mathrm{C} 2-\mathrm{C} 3-\mathrm{N} 2-\mathrm{C} 4$ & $-56.8(3)$ \\
\hline $\mathrm{C} 12-\mathrm{C} 6-\mathrm{C} 11-\mathrm{C} 10$ & $179.0(2)$ & $\mathrm{C} 5-\mathrm{C} 4-\mathrm{N} 2-\mathrm{C} 3$ & $56.1(3)$ \\
\hline
\end{tabular}

Hydrogen-bond geometry $\left(A,{ }^{\circ}\right)$

$\mathrm{Cg} 2$ is the centroid of the C6-C11 ring.

\begin{tabular}{lllll}
\hline$D-\mathrm{H} \cdots A$ & $D-\mathrm{H}$ & $\mathrm{H} \cdots A$ & $D \cdots A$ & $D-\mathrm{H}^{\cdots} A A$ \\
\hline $\mathrm{C} 3-\mathrm{H} 3 A \cdots \mathrm{O} 2^{\mathrm{i}}$ & 0.97 & 2.56 & $3.272(3)$ & 130 \\
$\mathrm{C} 5-\mathrm{H} 5 A \cdots \mathrm{O} 1^{\mathrm{ii}}$ & 0.97 & 2.56 & $3.469(3)$ & 156 \\
$\mathrm{~N} 2-\mathrm{H} 21 \cdots \mathrm{O} 2^{\mathrm{ii}}$ & $0.87(2)$ & $1.83(2)$ & $2.696(3)$ & $172(3)$
\end{tabular}


$\mathrm{N} 2-\mathrm{H} 22 \cdots \mathrm{O} 1^{\mathrm{iii}}$

$\mathrm{C} 4-\mathrm{H} 4 B \cdots C g 2^{\text {iv }}$

Symmetry codes: (i) $x, y+1, z$; (ii) $-x+2,-y+1,-z+1$; (iii) $-x+1,-y+1,-z+1$; (iv) $-x,-y,-z$.
$2.700(3)$

$3.473(3)$
$172(3)$

152 